RECEIVED

OCT 3 o 1996

OSTI

\title{
Inventory of LWR Spent Nuclear Fuel in the 324 Building
}

U.P. Jenquin

September 1996

Prepared for the U.S. Department of Energy under Contract DE-AC06-76RLO 1830

Pacific Northwest National Laboratory Operated for the U.S. Department of Energy by Battelle 


\title{
DISCLAIMER
}

This report was prepared as an account of work sponsored by an agency of the United States Government. Neither the United States Government nor any agency thereof, nor Battelle Memorial Institute, nor any of their employees, makes any warranty, express or implied, or assumes any legal liability or responsibility for the accuracy, completeness, or usefulness of any information, apparatus, product, or process disclosed, or represents that its use would not infringe privately owned rights. Reference herein to any specific commercial product, process, or service by trade name, trademark, manufacturer, or otherwise does not necessarily constitute or imply its endorsement, recommendation, or favoring by the United States Government or any agency thereof, or Battelle Memorial Institute. The views and opinions of authors expressed herein do not necessarily state or reflect those of the United States Government or any agency thereof.

\author{
PACIFIC NORTHWEST NATIONAL LABORATORY \\ operated by \\ BATTELLE \\ for the \\ UNITED STATES DEPARTMENT OF ENERGY \\ under Contract DE-ACO6 ?6RLO 1830
}

Printed in the United States of America

Available to DOE and DOE contractors from the

Office of Scientific and Technical Information, P.O. Box 62, Oak Ridge, TN 37831.

prices available from (615) $576-8401$.

Available to the public from the National Technical Information Service,

U.S. Department of Commerce, 5285 Port Royal Rd., Springfield, VA 22161 
Inventory of LWR Spent Nuclear Fuel in the 324 Building

U. P. Jenquin

September 1996

Prepared for

the U.S. Department of Energy

under Contract DE-AC06-76RLO 1830

Pacific Northwest National Laboratory

Richland, Washington 99352 


\section{DISCLAIMER}

This report was prepared as an account of work sponsored by an agency of the United States Government. Neither the United States Government nor any agency thereof, nor any of their employees, makes any warranty, express or implied, or assumes any legal liability or responsibility for the accuracy, completeness, or usefulness of any information, apparatus, product, or process disclosed, or represents that its use would not infringe privately owned rights. Reference herein to any specific commercial product, process, or service by trade name, trademark, manufacturer, or otherwise does not necessarily constitute or imply its endorsement, recommendation, or favoring by the United States Government or any agency thereof. The views and opinions of authors expressed herein do not necessarily state or reflect those of the United States Government or any agency thereof. 


\section{DISCLAIMER}

Portions of this document may be illegible in electronic image products. Images are produced from the best available original document. 


\section{Summary}

This document contains the results of calculations to estimate the decay heat, neutron source term, photon source term, and radioactive inventory of light-water-reactor spent nuclear fuel in the 324 Building at Pacific Northwest National Laboratory. 


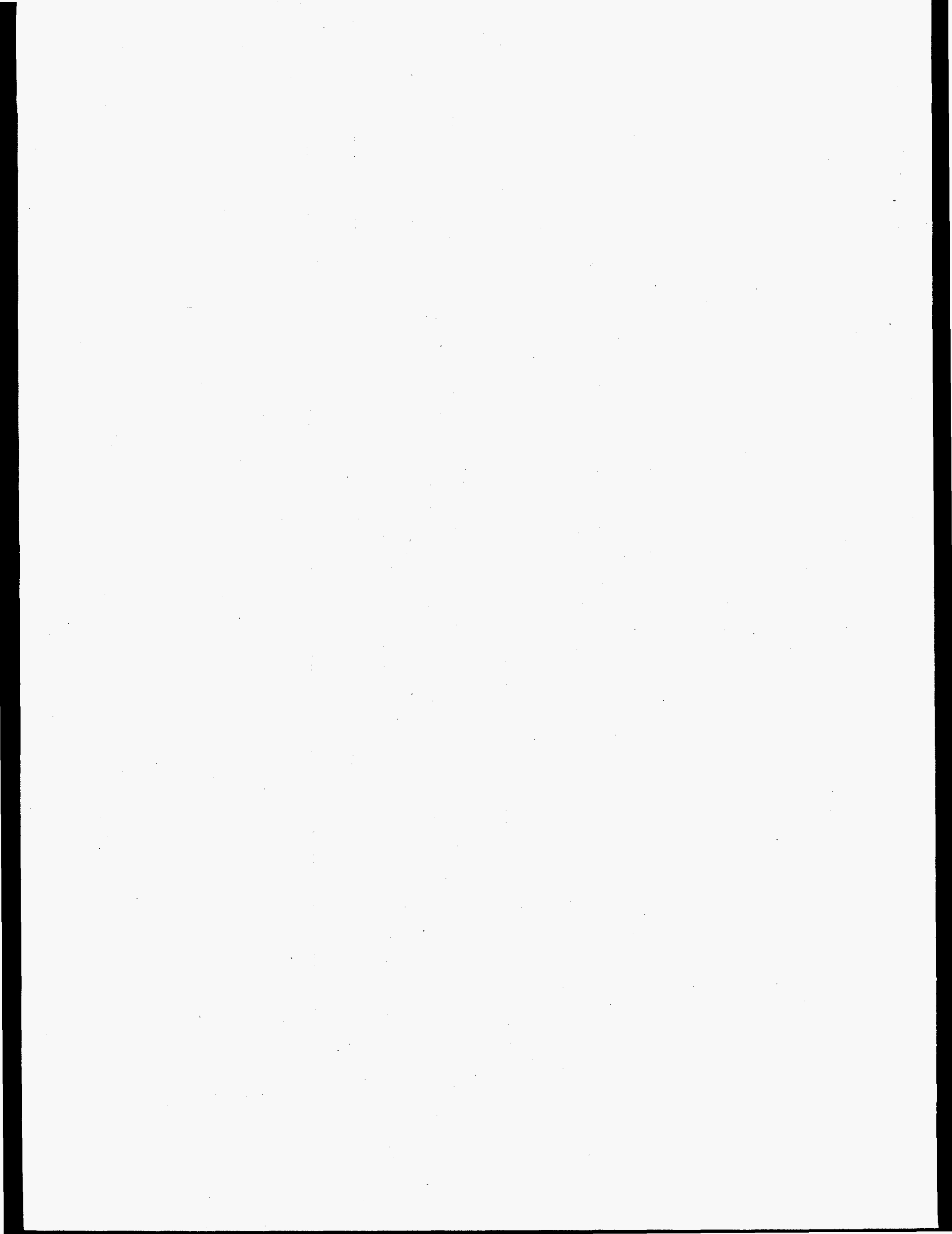




\section{Contents}

Summary $\ldots \ldots \ldots \ldots \ldots \ldots \ldots \ldots \ldots \ldots \ldots \ldots \ldots \ldots \ldots \ldots \ldots \ldots \ldots \ldots \ldots$ iii

1.0 Introduction $\ldots \ldots \ldots \ldots \ldots \ldots \ldots \ldots \ldots \ldots \ldots \ldots \ldots \ldots \ldots \ldots$

2.0 Description of Fuel $\ldots \ldots \ldots \ldots \ldots \ldots \ldots \ldots \ldots \ldots \ldots \ldots \ldots \ldots \ldots \ldots$

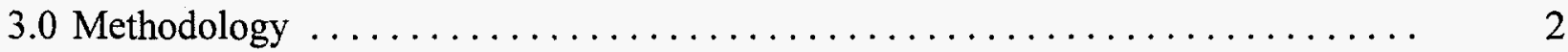

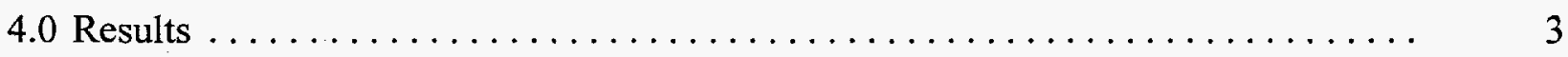

5.0 References $\ldots \ldots \ldots \ldots \ldots \ldots \ldots \ldots \ldots \ldots \ldots \ldots \ldots \ldots \ldots \ldots \ldots \ldots$

Appendix A - ORIGEN2 Input Files $\ldots \ldots \ldots \ldots \ldots \ldots \ldots \ldots \ldots \ldots \ldots \ldots$

Appendix B - Decay Heat and Source Terms $\ldots \ldots \ldots \ldots \ldots \ldots \ldots \ldots \ldots$

Appendix C - Radioactivity and Mass Inventories $\ldots \ldots \ldots \ldots \ldots \ldots \ldots \ldots \ldots \ldots$ 


\author{
Inventory of LWR Spent Nuclear Fuel in the 324 Building \\ September, 1996 \\ Urban Jenquin \\ Materials and Engineering Analysis Group \\ Engineering and Analytic Sciences Department
}

\title{
1.0 Introduction
}

Irradiated light-water-reactor (LWR) fuel was brought into the 324 building for research purposes during the 1980's. The fuel consists of three complete Point Beach assemblies, two partial Calvert Cliffs assemblies, one complete Cooper assembly, one partial Cooper assembly, loose full-length fuel rods, segments of fuel rods, and powder. The Cooper Reactor is a boiling water reactor (BWR) and the Calvert Cliffs and Point Beach Reactors are pressurized water reactors (PWRs). One of the Calvert Cliffs assemblies contains some of its original fuel rods plus fuel rods from another assembly. All of the fuel rods in the Cooper assemblies plus fulllength fuel rods which have been removed from the Calvert Cliffs assemblies will be consolidated in one canister. Each of the remaining assemblies will be placed in separate canisters.

This document contains the results of calculations to estimate the radioactive inventory of each canister. The calculations also give the decay heat, neutron source term, and photon source term for each canister. The source terms are needed for dose rate assessments and the inventories are needed to evaluate accident scenarios. Partial-length fuel rods and powder are not included in the estimates.

\subsection{Description of Fuel}

The characteristics of the fuel are summarized in Table 1. The Approved Testing Material (ATM) No. is a number assigned to various types of fuel by the Materials Characterization Center (MCC). ATM-108 fuel is Cooper fuel which contains gadolinium burnable poison. Initially, there were 10 fuel rods containing gadolinium. One was segmented, so nine remain intact. ATM-105 is the remainder of the Cooper fuel. Calvert Cliffs Assembly D047 contains 126 of its original 176 fuel rods plus 13 fuel rods from another assembly (BT03); hence, two separate columns are used to describe the fuel. The three Point Beach assemblies (H07, H12, and $\mathrm{H} 25$ ) are similar to each other.

The Cooper assemblies consist of fuel rods with several enrichments ranging from $1.33 \mathrm{wt} \%$ to $2.93 \mathrm{wt} \%$. The assembly-average enrichment is $2.50 \mathrm{wt} \%$, as shown in Table 1 . The number of fuel rods in each assembly as of June 1996 is given on the third-to-the-last line of Table 1 . The number of loose, full-length fuel rods are in addition to those in the assembly. Current plans are to remove all of the Cooper fuel rods from Assemblies CZ346 and CZ348 and consolidate them 
with the other loose Cooper fuel rods and loose Calvert Cliffs fuel rods into one canister. The consolidated fuel rods canister will contain 112 full-length fuel rods.

Table 1. Characteristics of LWR Spent Fuel in 324 Building

\begin{tabular}{|l|c|c|c|c|c|c|}
\hline Assembly ID & D101 & \multicolumn{2}{|c|}{ D047 } & CZ346 & CZ348 & H07* \\
\hline Reactor & $\begin{array}{c}\text { Calvert } \\
\text { Cliffs }\end{array}$ & \multicolumn{2}{|c|}{ Calvert Cliffs } & Cooper & Cooper & $\begin{array}{c}\text { Point } \\
\text { Beach }\end{array}$ \\
\hline ATM No. & ATM-103 & ATM-104 & ATM-106 & ATM-105/108 & ATM-105/108 & NA \\
\hline Initial Enrichment, wt\% & 2.72 & 3.04 & 2.45 & 2.50 & 2.50 & 3.19 \\
\hline Burnup, GWD/MTU & 30.7 & 41.8 & 42.7 & 28.05 & 27.48 & 32.9 \\
\hline Discharge Date & $10 / 18 / 80$ & $04 / 17 / 82$ & $10 / 18 / 80$ & $05 / 21 / 82$ & $05 / 21 / 82$ & $10 / 09 / 81$ \\
\hline Decay Time, yr & 15.7 & 14.2 & 15.7 & 14.1 & 14.1 & 14.7 \\
\hline Fuel Rod Mass, KgU & 2.215 & 2.215 & 2.244 & 3.89 & 3.89 & 2.241 \\
\hline $\begin{array}{l}\text { No. of Fuel Rods in } \\
\text { Assembly }\end{array}$ & 168 & 126 & 13 & 37 & 49 & 179 \\
\hline Assembly Mass, KgU & 372.1 & 279.1 & 29.2 & 143.9 & 190.6 & 401.1 \\
\hline No. of Loose Fuel Rods & 6 & 7 & 4 & 9 & 0 & 0 \\
\hline
\end{tabular}

* Assemblies $\mathrm{H} 12$ and $\mathrm{H} 25$ have the same information as Assembly $\mathrm{H} 07$.

\subsection{Methodology}

Extensive characterization ${ }^{(1-4)}$ of the Calvert Cliffs and Cooper fuel was made by the Materials Characterization Center (MCC) at the Pacific Northwest Laboratory. The characterization included comparison of measured data to ORIGEN2 ${ }^{(5-7)}$ calculated values made at Quality Assurance Impact Level 1. The correlations show quite good agreement between calculated and measured isotopic quantities. However, the calculated-to-measured value for the activity of $\mathrm{Cm}-243 / 244$ typically was $6 \%$ low, a non-conservative result. $\mathrm{Cm}-244$ is the dominant neutron source. The same calculational methods were used for this analysis. The ORIGEN2 code utilized Pacific Northwest National Laboratory's software quality assurance process as defined in the Software Control Procedures (SCPs). The SCP process is an American Society of Mechanical Engineers NQA-1 based program, and has been tested and reviewed in accordance with the appropriate SCP procedural requirements.

The ORIGEN2 models developed under the MCC Project were used to calculate inventories, decay heat, and source terms. Under the MCC Project, detailed irradiation histories were developed for each assembly except for Assembly CZ348. Cycle-average power densities were developed for Assembly CZ348. The calculations were made on an assembly-average basis. 
Available information ${ }^{(8)}$ indicates that all three Point Beach assemblies underwent similar irradiation; therefore, a single ORIGEN2 calculation provides the inventories, decay heat, and source terms. Detailed irradiation histories are not available for the Point Beach assemblies. Fuel cycle information in Reference 8 was used to develop cycle-average power densities for the Point Beach fuel. Detailed within-cycle power history has a negligible effect on the results. The ORIGEN2 input files are listed in Appendix A.

During irradiation the axial power distribution is peaked toward the center of the fuel rod. Therefore, the burnup in the central portion of the fuel rod is more than the average burnup and the burnup near the ends of the fuel rod is less than the average burnup. For many considerations the effect of nonuniform burnup is very small and need not be addressed in detail. The buildup of transplutonium isotopes is quite sensitive to nonuniform burnup. It is increasingly important as the number of neutron captures increases. The quantity of transplutonium isotopes is small compared to the residual uranium; however, $\mathrm{Cm}-244$ is the dominant neutron source. Therefore, supplemental ORIGEN2 calculations were made to determine an axial shape for the neutron source and to determine a correction factor for the total neutron source term. Several axial Cs-137 spectral gamma scans were made from ATM-104 fuel rods ${ }^{(2)}$. These gamma scans closely approximate the axial burnup distribution. A typical gamma scan was used to estimate the peak-to-average value of about 1.09. Consequently, the localized peak burnup is assumed to be $9 \%$ greater than the average for the assembly.

Inconel grid spacers and stainless steel end fittings contain cobalt as an impurity. During irradiation, radioactive Co-60 is formed. Each Co-60 decay results in two photons, one with 1.17 $\mathrm{MeV}$ of energy and one with $1.33 \mathrm{MeV}$ of energy. The Co-60 activity in the stainless steel end fittings was estimated based on measured values for a burnable poison rod assembly in Point Beach fuel ${ }^{(9)}$, and making corrections for decay time and fuel burnup. The Co-60 activity in the grid spacers was calculated with ORIGEN2 assuming they receive assembly-average neutron fluence. Based on a tabulation of PWR hardware information ${ }^{(10)}$, assumptions were made which maximize the Co-60 activity. The consolidated canister does not contain end fittings or grid spacers, so the Co-60 activity is expected to be negligible in this canister.

\subsection{Results}

Calculated neutron and photon total source terms and decay heat values for each assembly decayed to June 1996 are given in Table 2. As expected, Assembly D047 has the highest neutron source term; thus, it is considered the worst case. Fission product photons dominate the photon source term in the fuel. The neutron source term is dominated by spontaneous fission of $\mathrm{Cm}-244$. Depending on the assembly, between 92 and $96 \%$ of the neutron source term is due to $\mathrm{Cm}-244$. Relative neutron and photon source values are also given in Table 2. Decay heat values and source term values are given in Appendix B for longer decay times.

The June 1996 photon source spectrum for the fuel in Assembly D047 is given in Table 3. The energies listed are mid-point values as used by ORIGEN2. The major contribution to the 0.575 $\mathrm{MeV}$ group is from decay of Cs-137. Photon source spectra for longer decay times are given in Appendix B. 
Table 2. Source Terms and Decay Heat for LWR Spent Fuel

\begin{tabular}{|c|c|c|c|c|c|}
\hline Assembly ID & D101 & D047 & $\mathrm{H} 07^{*}$ & Consolidated & Total \\
\hline $\begin{array}{l}\text { No. of Fuel } \\
\text { Rods in } \\
\text { Canister }\end{array}$ & 168 & 139 & 179 & 112 & 956 \\
\hline $\begin{array}{l}\text { Decay Heat } \\
\text { Rate, watts }\end{array}$ & 328.0 & 407.0 & 390.7 & 337.1 & 2,244 \\
\hline $\begin{array}{l}\text { Neutron } \\
\text { Source, } \\
\mathrm{n} / \mathrm{sec}^{* *}\end{array}$ & $6.75 \mathrm{E}+07$ & $1.84 \mathrm{E}+08$ & $6.66 \mathrm{E}+07$ & $7.54 \mathrm{E}+07$ & $6.32 E+08$ \\
\hline $\begin{array}{l}\text { Relative } \\
\text { Neutron } \\
\text { Source }\end{array}$ & 0.367 & 1.000 & 0.362 & 0.410 & NA \\
\hline \multicolumn{6}{|c|}{ Photon Source, gammas $/ \mathrm{sec}$} \\
\hline Fuel & $2.05 E+15$ & $2.36 \mathrm{E}+15$ & $2.41 E+15$ & $2.04 \mathrm{E}+15$ & $1.37 \mathrm{E}+16$ \\
\hline Grid Spacers & $8.35 \mathrm{E}+12$ & $1.23 \mathrm{E}+13$ & $5.83 \mathrm{E}+13$ & 0 & $1.95 \mathrm{E}+14$ \\
\hline Top Nozzle & & & $9.93 E+11$ & 0 & $2.98 \mathrm{E}+12$ \\
\hline $\begin{array}{l}\text { Bottom } \\
\text { Nozzle }\end{array}$ & $6.88 \mathrm{E}+11$ & $1.14 \mathrm{E}+12$ & $8.39 \mathrm{E}+11$ & 0 & $4.34 \mathrm{E}+12$ \\
\hline \multicolumn{6}{|c|}{ Relative Photon Source } \\
\hline Fuel & 0.870 & 1.000 & 1.023 & 0.867 & NA \\
\hline Grid Spacers & 0.143 & 0.210 & 1.000 & 0 & NA \\
\hline Top Nozzle & & & 1.000 & 0 & NA \\
\hline $\begin{array}{l}\text { Bottom } \\
\text { Nozzle }\end{array}$ & 0.603 & 1.000 & 0.736 & 0 & NA \\
\hline
\end{tabular}

* Same information for assemblies H-12 and H-25.

** Canister neutron source is increased by $6 \%$ to compensate for underpredicting $\mathrm{Cm}-243 / 244$ activity. Total neutron source is increased by $20 \%$ to account for detailed axial shape vs. assembly average. 
Table 3. Calculated Photon Source for Assembly D047

\begin{tabular}{|c|c|}
\hline E, MeV & Photons/sec \\
\hline 0.010 & $6.00 \mathrm{E}+14$ \\
\hline 0.025 & $1.21 \mathrm{E}+14$ \\
\hline 0.0375 & $1.58 \mathrm{E}+14$ \\
\hline 0.0575 & $1.23 \mathrm{E}+14$ \\
\hline 0.085 & $6.84 \mathrm{E}+13$ \\
\hline 0.125 & $6.62 \mathrm{E}+13$ \\
\hline 0.225 & $5.70 \mathrm{E}+13$ \\
\hline 0.375 & $2.43 \mathrm{E}+13$ \\
\hline 0.575 & $1.06 \mathrm{E}+15$ \\
\hline 0.85 & $4.68 \mathrm{E}+13$ \\
\hline 1.25 & $3.25 \mathrm{E}+13$ \\
\hline 1.75 & $9.98 \mathrm{E}+11$ \\
\hline 2.25 & $6.88 \mathrm{E}+08$ \\
\hline 2.75 & $2.89 \mathrm{E}+08$ \\
\hline 3.5 & $2.62 \mathrm{E}+07$ \\
\hline 5.0 & $7.46 \mathrm{E}+06$ \\
\hline 7.0 & $8.61 \mathrm{E}+05$ \\
\hline 9.5 & $9.89 \mathrm{E}+04$ \\
\hline \hline Total & $2.36 \mathrm{E}+15$ \\
\hline & \\
\hline
\end{tabular}

The axial neutron source distribution for Assembly D047 is given in Table 4. The axial distribution for the other canisters is expected to be approximately the same.

Calculated inventories for selected actinides and fission products, and for Co-60 in the grid spacers are given in Appendix C. As expected, Calvert Cliffs Assembly D047 has the most Cm244 , the dominant contributor to the neutron source. The dominant contributor to the photon source, Cs-137, is 1\% higher in the Point Beach assemblies than in Assembly D047. Except for Co-60, no estimate of radioactive activation products was made because they are insignificant compared to actinides and fission products. Mass inventories are given in Appendix $\mathrm{C}$ for the isotopes corresponding to the list in the curie tables. 
Table 4. Axial Neutron Source Distribution

\begin{tabular}{|c|c|}
\hline $\begin{array}{c}\text { Dist. From } \\
\text { Bottom of } \\
\text { Fuel, cm }\end{array}$ & $\begin{array}{c}\text { Relative } \\
\text { Source }\end{array}$ \\
\hline \hline 0.0 & 0.026 \\
\hline 3.4 & 0.026 \\
\hline 6.9 & 0.055 \\
\hline 10.3 & 0.108 \\
\hline 13.8 & 0.200 \\
\hline 20.7 & 0.342 \\
\hline 27.6 & 0.547 \\
\hline 34.5 & 0.677 \\
\hline 44.8 & 0.827 \\
\hline 62.1 & 1.000 \\
\hline 280.0 & 1.000 \\
\hline 300.7 & 0.827 \\
\hline 311.1 & 0.677 \\
\hline 321.4 & 0.436 \\
\hline 330.0 & 0.264 \\
\hline 335.2 & 0.148 \\
\hline 338.7 & 0.077 \\
\hline 343.8 & 0.038 \\
\hline 349.0 & 0.038 \\
\hline Average & $\mathbf{0 . 8 2 8}$ \\
\hline & \\
\hline
\end{tabular}




\subsection{References}

1. Guenther, R. J., D. E. Blahnik, T. K. Campbell, U. P. Jenquin, J. E. Mendel, L. E. Thomas, and C. K. Thornhill. April 1988. "Characterization of Spent Fuel Approved Testing Material - ATM-103". PNL-5109-103, Pacific Northwest Laboratory, Richland, Washington.

2. Guenther, R. J., D. E. Blahnik, T. K. Campbell, U. P. Jenquin, J. E. Mendel, L. E. Thomas, and C. K. Thornhill. December 1991. "Characterization of Spent Fuel Approved Testing Material - ATM-104". PNL-5109-104, Pacific Northwest Laboratory, Richland, Washington.

3. Guenther, R. J., D. E. Blahnik, T. K. Campbell, U. P. Jenquin, J. E. Mendel, and C. K. Thornhill. October 1988. "Characterization of Spent Fuel Approved Testing Material ATM-106". PNL-5109-106, Pacific Northwest Laboratory, Richland, Washington.

4. Guenther, R. J., D. E. Blahnik, T. K. Campbell, U. P. Jenquin, J. E. Mendel, L. E. Thomas, and C. K. Thornhill. December 1991. "Characterization of Spent Fuel Approved Testing Material - ATM-105". PNL-5109-105, Pacific Northwest Laboratory, Richland, Washington.

5. Croff, A. G. July 1980. "ORIGEN2 - A Revised and Updated Version of the Oak Ridge Isotope Generation and Depletion Code". ORNL-5621, Oak Ridge National Laboratory, Oak Ridge, Tennessee.

6. Croff, A. G. July 1980. "A User's Manual for the ORIGEN2 Computer Code". ORNL/TM-7175, Oak Ridge National Laboratory, Oak Ridge, Tennessee.

7. Croff, A. G., M. A. Bjerke, G. W. Morrison, and L. M. Petrie. September 1978. "Revised Uranium-Plutonium Cycle PWR and BWR Models for the ORIGEN Computer Code". ORNL/TM-6051, Oak Ridge National Laboratory, Oak Ridge, Tennessee.

8. 1986. "Point Beach Nuclear Plant Unit \#1 Fuel Assembly Record for Assemblies H07, H12, and H25". Wisconsin Michigan Power Company, Milwaukee, Wisconsin.

9. Migliore, R. J., B. D. Reid, S. K. Fadeff, K. A. Pauley, and U. P. Jenquin. September 1994. "Non-Fuel Assembly Components: 10 CFR 61.55 Classification for Waste Disposal". PNL10103, Pacific Northwest Laboratory, Richland, Washington.

10. September 1986. "Nuclear Fuel Data". WTSD-TME-148, Westinghouse Electric Corp., Madison, Pennsylvania. 
Appendix A

ORIGEN2 Input Files 
-1
-1

-1
-1

TIT PWR Calvert Cliffs - ATM-103 Fuel E=2.72 30.7 GWD $/ \mathrm{MTU}$

BAS

MTU

LIB

PHO

LIP

INP

RDA

BUP

IRP

IRP

IRP

DEC

IRP

IRP

IRP

IRP

DEC

IRP

DEC

IRP

IRP

IRP

IRP

BUP

BAS

RDA

MOV

DEC

DEC

DEC

DEC

DEC

DEC

$D E C$

HED

HED

HED

HED

HED

OPTA

OPTL

OPTF

CUT

OUT

STP

2

4

4

4

0

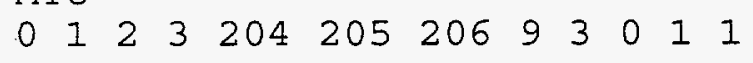

10110210310

000

$\begin{array}{llllll}1 & 1 & -1 & -1 & 1 & 1\end{array}$

Burnup to $30,700 \mathrm{MWD} / \mathrm{MTU}$

100.0

200.0

33.06

33.06

306.0

33.06

377.0

398.1

25.97

417.6

18.12

527.6

29.37

646.0

29.37

671.0

759.0

29.37

840.0

$959.1 \quad 25.30$

$1080.9 \quad 12.50$

$1190.9 \quad 26.01$

$1306.0 \quad 26.01$

$\begin{array}{llll}1 & 2 & 4 & 2\end{array}$

$\begin{array}{llll}2 & 3 & 4 & 0\end{array}$

3440

$\begin{array}{llll}4 & 5 & 4 & 0\end{array}$

$5 \quad 6 \quad 4 \quad 0$

$\begin{array}{llll}6 & 7 & 4 & 0\end{array}$

$\begin{array}{llll}7 & 8 & 4 & 0\end{array}$

8940

$\begin{array}{llll}9 & 1 & 4 & 0\end{array}$

$\begin{array}{llll}1 & 2 & 4 & 0\end{array}$

$\begin{array}{llll}2 & 3 & 4 & 0\end{array}$

$\begin{array}{llll}3 & 4 & 4 & 0\end{array}$

$\begin{array}{llll}4 & 5 & 4 & 0\end{array}$

$\begin{array}{llll}5 & 6 & 4 & 0 \\ 6 & 7 & 4 & 0\end{array}$

168 Rods

2. $215 \mathrm{KgU} / \mathrm{Rod}$

$\begin{array}{llll}7 & 7 & 0 & .3721\end{array}$

1.

5.

15.7

19.7

29.7

49.7

119.7

1 June 1996

$\begin{array}{llll}7 & 8 & 5 & 2\end{array}$

8950

$\begin{array}{llll}9 & 1 & 5 & 0\end{array}$

$\begin{array}{llll}1 & 2 & 5 & 0\end{array}$

$\begin{array}{llll}2 & 3 & 5 & 0\end{array}$

2 June 2000

3 June 2010

4 June 2030

5 June 2100

$\begin{array}{llllllllllllllllllllllll}8 & 8 & 8 & 8 & 7 & 8 & 7 & 8 & 7 & 8 & 8 & 8 & 8 & 8 & 8 & 8 & 8 & 8 & 8 & 8 & 8 & 8 & 8 & 8\end{array}$

$\begin{array}{llllllllllllllllllllllll}8 & 8 & 8 & 8 & 7 & 8 & 7 & 8 & 7 & 8 & 8 & 8 & 8 & 8 & 8 & 8 & 8 & 8 & 8 & 8 & 8 & 8 & 8 & 8\end{array}$

$\begin{array}{llllllllllllllllllllllll}8 & 8 & 8 & 8 & 7 & 8 & 7 & 8 & 7 & 8 & 8 & 8 & 8 & 8 & 8 & 8 & 8 & 8 & 8 & 8 & 8 & 8 & 8 & 8\end{array}$

$\begin{array}{llllll}5 & 1.0-5 & 7 & 1.0-10 & -1\end{array}$

5100

4

80000134430.6000013 .6

$\begin{array}{rlrl}922380 & 972580 . & 0 & 0.0 \\ 70000 & 27.2 & 90000 & 5.7 \\ 470000 & 1.1 & 200000 & 45.4 \\ 280000 & 28.4 & 270000 & 23.2\end{array}$

26000059.0 14000045.4

END 
$-1$

$-1$

TIT

PWR Calvert Cliffs - ATM-104 Fuel E=3.038 41.8 GWD/MTU

BAS

MTU

IIB

$\begin{array}{llllllllllll}0 & 1 & 2 & 3 & 204 & 205 & 206 & 9 & 3 & 0 & 1 & 1\end{array}$

$\mathrm{PHO}$

$\begin{array}{llll}101 & 102 & 103 \quad 10\end{array}$

LIP

$\begin{array}{lll}0 & 0 & 0\end{array}$

INP

$\begin{array}{llllll}1 & 1 & -1 & -1 & 1 & 1\end{array}$

RDA

IRP

IRP

IRP

DEC

IRP

IRP

IRP

IRP

DEC

IRP

DEC

IRP

IRP

IRP

IRP

DEC

IRP

IRP

IRP

IRP

BUP

BAS

RDA

MOV

$D E C$

DEC

DEC

$\mathrm{DEC}$

DEC

DEC

DEC

HED

HED

HED

HED

HED

OPTL

OPTA

OPTF

CUT

OUT

STP

Burnup to $41,800 \mathrm{MWD} / \mathrm{MTU}$

$\begin{array}{rlllll}100.0 & 32.83 & 1 & 2 & 4 & 2 \\ 200.0 & 32.83 & 2 & 3 & 4 & 0 \\ 306.0 & 32.83 & 3 & 4 & 4 & 0 \\ 377.0 & & 4 & 5 & 4 & 0 \\ 398.9 & 30.72 & 5 & 6 & 4 & 0 \\ 418.3 & 20.38 & 6 & 7 & 4 & 0 \\ 528.3 & 31.10 & 7 & 8 & 4 & 0 \\ 646.0 & 31.10 & 8 & 9 & 4 & 0 \\ 671.0 & & 9 & 1 & 4 & 0 \\ 759.0 & 31.10 & 1 & 2 & 4 & 0 \\ 840.0 & & 2 & 3 & 4 & 0 \\ 958.3 & 27.23 & 3 & 4 & 4 & 0 \\ 1080.1 & 13.41 & 4 & 5 & 4 & 0 \\ 1190.1 & 27.21 & 5 & 6 & 4 & 0 \\ 1306.0 & 27.21 & 6 & 7 & 4 & 0 \\ 1391.0 & & 7 & 8 & 4 & 0 \\ 1506.0 & 21.40 & 8 & 9 & 4 & 0 \\ 1621.0 & 21.40 & 9 & 1 & 4 & 0 \\ 1736.0 & 21.40 & 1 & 2 & 4 & 0 \\ 1852.0 & 21.40 & 2 & 3 & 4 & 0\end{array}$

126 Rods

$2.215 \mathrm{KgU} / \mathrm{Rod}$

$$
\begin{array}{llll}
3 & 3 & 0 & .2790
\end{array}
$$

1 .

5 .

14.2

18.2

28.2

48.2

118.2

1 June 1996

2 June 2000

3 June 2010

4 June 2030

5 June 2100

2

$\begin{array}{llllllllllllllllllllllll}8 & 8 & 8 & 8 & 7 & 8 & 7 & 8 & 7 & 8 & 8 & 8 & 8 & 8 & 8 & 8 & 8 & 8 & 8 & 8 & 8 & 8 & 8 & 8\end{array}$

$\begin{array}{llllllllllllllllllllllll}8 & 8 & 8 & 8 & 7 & 8 & 7 & 8 & 7 & 8 & 8 & 8 & 8 & 8 & 8 & 8 & 8 & 8 & 8 & 8 & 8 & 8 & 8 & 8\end{array}$

$\begin{array}{llllllllllllllllllllllll}8 & 8 & 8 & 8 & 7 & 8 & 7 & 8 & 7 & 8 & 8 & 8 & 8 & 8 & 8 & 8 & 8 & 8 & 8 & 8 & 8 & 8 & 8 & 8\end{array}$

$51.0-5 \cdot 71.0-10-1$

$\begin{array}{llll}5 & 1 & 0 & 0\end{array}$ 4

922340246.92235030380 .922380969370 .000 


$\begin{array}{lllllllll}4 & 80000 & 134520 & 60000 & 20.4 & 70000 & 26.1 & 90000 & 5.7 \\ 4 & 170000 & 5.7 & 260000 & 51.1 & 470000 & 1.1 & 200000 & 36.3 \\ 4 & 130000 & 36.3 & 140000 & 36.3 & 280000 & 28.4 & 270000 & 30.9 \\ 0 & & & & & & & \end{array}$

END 


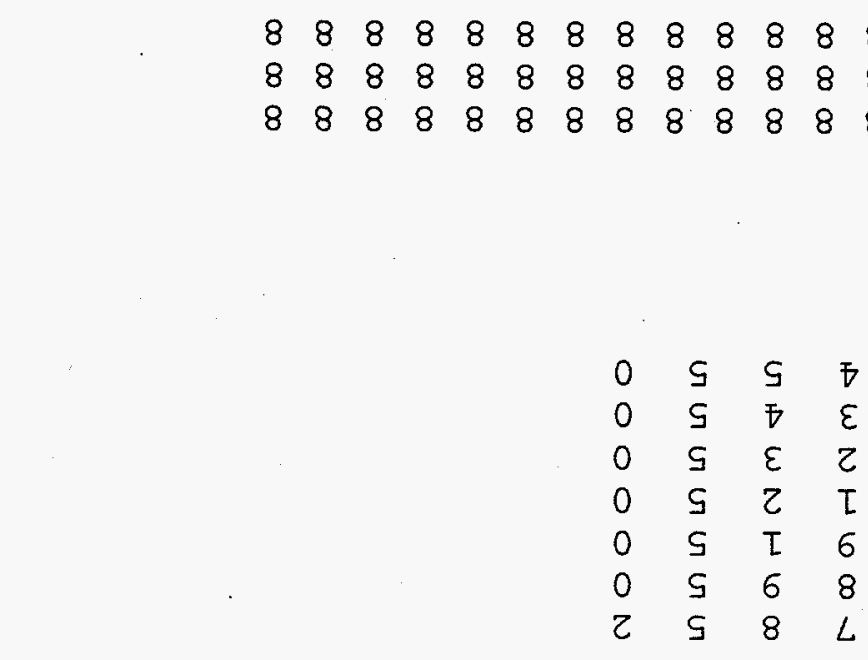

$\begin{array}{llllllllllll}8 & 8 & 8 & L & 8 & L & 8 & L & 8 & 8 & 8 & 8\end{array}$ $\begin{array}{llllllllllll}8 & 8 & 8 & L & 8 & L & 8 & L & 8 & 8 & 8 & 8\end{array}$ $\begin{array}{lll}8 & 8\end{array}$

$\begin{array}{lllllll}L & 8 & L & 8 & 8 & 8 & 8\end{array}$

\begin{tabular}{|c|c|c|}
\hline & 千 & $L$ \\
\hline & ₹ & 9 \\
\hline & 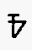 & 与 \\
\hline 0 & 7 & t \\
\hline 0 & † & $\varepsilon$ \\
\hline 0 & $\varpi$ & z \\
\hline 0 & † & $\tau$ \\
\hline 0 & $\varpi$ & 6 \\
\hline 0 & $\varpi$ & 8 \\
\hline 0 & $\mp$ & $L$ \\
\hline 0 & $\varpi$ & 9 \\
\hline 0 & $\varpi$ & $\varsigma$ \\
\hline 0 & 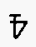 & $\overline{7}$ \\
\hline 0 & $\varpi$ & $\varepsilon$ \\
\hline 0 & 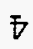 & $\tau$ \\
\hline 0 & ‡ & $\tau$ \\
\hline 0 & $\varpi$ & 6 \\
\hline 0 & $\Phi$ & 8 \\
\hline 0 & $\varpi$ & $L$ \\
\hline 0 & $\varpi$ & 9 \\
\hline 0 & $\bar{\tau}$ & $\mathcal{G}$ \\
\hline 0 & $\varpi$ & ఫ \\
\hline 0 & $\varpi$ & $\varepsilon$ \\
\hline & $\varpi$ & $\tau$ \\
\hline
\end{tabular}

\section{$\mathrm{\Lambda LW} / \triangle M W$}

$$
\text { . }
$$$$
\tau \quad I 0 \varepsilon
$$
ootz əun $S$

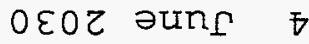
oIOZ әun $\Omega$ $000 z$ әun $\Omega$ 966I วun $\tau$ $L \cdot 6 \tau \tau$ $L \cdot 67$ $L \cdot 62$ $L \cdot 6 \tau$ $L \cdot S T$ $\cdot \mathrm{s}$$$
\cdot \tau
$$$$
2620
$$$$
\begin{array}{r}
\text { poy } / \cap 6 y \text { चெ乙 } 2 \\
\text { spoy } \varepsilon I
\end{array}
$$
spoy $\varepsilon I$

$$
\begin{aligned}
& \varepsilon S \cdot \tau Z \\
& \varepsilon S \cdot \tau Z \\
& \tau \bar{\tau} \cdot 6 \\
& \tau g \cdot \sigma \tau
\end{aligned}
$$$$
0^{\circ} \angle \tau \tau z
$$$$
9^{\circ} \text {. } 2002
$$$$
9^{\circ} \mathrm{I68I}
$$$$
L \cdot \angle 9 L T
$$$$
L \tau \cdot \tau Z
$$$$
\text { 0. IS9I }
$$$$
67 \cdot 6 I
$$$$
67^{\circ} \cdot 6 I
$$$$
\text { I0. } 6
$$$$
\angle 8^{\circ} 9 \text { I }
$$$$
0.0 \angle S T
$$$$
0.28 D T
$$$$
0 \cdot \angle S E T
$$$$
\varepsilon \cdot 8 \succsim \varepsilon \tau
$$$$
\varepsilon \cdot 8 \varepsilon ट \tau
$$$$
0 \cdot \varepsilon \tau \zeta \tau
$$$$
0.88 I \tau
$$$$
\text { โゅ・๑乙 }
$$$$
\text { โォ.ง乙 }
$$$$
0^{\circ} \angle I I L
$$$$
0^{\circ} \text { IIOT }
$$$$
\text { โ五 ऽ乙 }
$$$$
0 \cdot \text { II6 }
$$$$
0^{\circ} \text { II8 }
$$

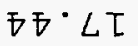$$
89^{\circ} 8 \mathrm{Z}
$$$$
0.0 \varepsilon L
$$$$
\text { L.999 }
$$$$
85^{\circ} 8 \mathrm{Z}
$$$$
0.885
$$$$
\varepsilon \sigma^{\circ} 6 z
$$$$
\varepsilon 6 \cdot 6 z
$$$$
\varepsilon \sigma^{\circ} 6 z
$$$$
\varepsilon 6^{\circ} 6 z
$$$$
0.867
$$$$
0.597
$$$$
0.87 \varepsilon
$$$$
0^{\circ} \text { 乙हट }
$$$$
0.9 \text { I }
$$

F山dO

$\forall$ IdO

TLdO

वज्ञH

$\square \mathrm{GH}$

$\square \mathrm{GH}$

$\mathrm{व} B \mathrm{H}$

$व$ वस

गजם

गHव

गदव

गद्ध一

ग马्व

गद्धव

गЕ

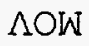

$\forall \square \mathcal{A}$

S甘G

dกg

dy I

dUI

dU्रI

dUI

ग马व

dUI

गีप

dUI

dUI

dyI

dUI

ग马व

dy I

dyI

dUI

गद्मव

ded

dyI

dUI

गसव

d\&I

dy I

dUI

dYI

dกg

$\forall \subset \mathcal{Y}$

dNI

dIT

OHd

OI EOT ZOL TOL

घIT

SHg

LIL 


\begin{tabular}{|c|c|c|c|c|c|c|c|c|}
\hline CUT & $1.0-5$ & 71.0 & 10 & & & & & \\
\hline OUT & 1 & 0 & & & & & & \\
\hline STP & 4 & & & & & & & \\
\hline 2 & 922340 & 199. & 922350 & 24530 . & 922380 & 975270. & 00.0 & \\
\hline 4 & 80000 & 134520 . & 60000 & 21.6 & 70000 & 49.9 & 90000 & 11.3 \\
\hline 4 & 170000 & 11.3 & 260000 & 51.1 & 470000 & 1.1 & 200000 & 45.4 \\
\hline 4 & 130000 & 45.4 & 140000 & 45.4 & 280000 & 28.4 & 00.0 & \\
\hline
\end{tabular}

END 
$-1$

TIT BWR Cooper - Assembly CZ346 - ATM-105/108 E=2.50 28.05 GWD/MTU BAS MTU

L.IB $\quad \begin{array}{lllllllllllll}0 & 1 & 2 & 3 & 251 & 252 & 253 & 9 & 3 & 0 & 1 & 4\end{array}$

PHO $\quad 101 \quad 102 \quad 103 \quad 10$

LIP $\quad 0 \quad 0 \quad 0$

INP $\quad \begin{array}{llllll}1 & 1 & -1 & -1 & 1 & 1\end{array}$

RDA Burnup to $28,050 \mathrm{MWD} / \mathrm{MTU}$

BUP

IRP

DEC

IRP

IRP

DEC

IRP

IRP

DEC

IRP

IRP

DEC

IRP

IRP

DEC

IRP

DEC

IRP

IRP

$D E C$

IRP

IRP

$D E C$

IRP

IRP

IRP

IRP

DEC

IRP

IRP

$19 \quad 15.63$

27

$90 \quad 14.21$

157

174

182

215

225

296

451

486

505

22.74

14.21

25.58

27.01

14.21

525

14.21

532

554

566

621

674

693

718

807

866

880

904

919

945

953

996

1001

IRP

1017

DEC

IRP

IRP

IRP

DEC

IRP

DEC

IRP

IRP

IRP

IRP

DEC

1022

1044

1083

1172

1203

1367

2166

2189

2214

2228

2326

2335

IRP

2377

25.58

22.74

22.74

19.89

19.89

22.74

15.46

20.98

11.04

20.99

20.99

8.84

20.99

19.88

13.25

17.67

16.95

10.33

11.48

5.74

10.91

10.91

$\begin{array}{llll}1 & 2 & 4 & 2 \\ 2 & 3 & 4 & 0 \\ 3 & 4 & 4 & 0 \\ 4 & 5 & 4 & 0 \\ 5 & 6 & 4 & 0 \\ 6 & 7 & 4 & 0 \\ 7 & 8 & 4 & 0 \\ 8 & 9 & 4 & 0 \\ 9 & 1 & 4 & 0 \\ 1 & 2 & 4 & 0 \\ 2 & 3 & 4 & 0 \\ 3 & 4 & 4 & 0 \\ 4 & 5 & 4 & 0 \\ 5 & 6 & 4 & 0 \\ 6 & 7 & 4 & 0 \\ 7 & 8 & 4 & 0 \\ 8 & 9 & 4 & 0 \\ 9 & 1 & 4 & 0 \\ 1 & 2 & 4 & 0 \\ 2 & 3 & 4 & 0 \\ 3 & 4 & 4 & 0 \\ 4 & 5 & 4 & 0 \\ 5 & 6 & 4 & 0 \\ 6 & 7 & 4 & 0 \\ 7 & 8 & 4 & 0 \\ 8 & 9 & 4 & 0 \\ 9 & 1 & 4 & 0 \\ 1 & 2 & 4 & 0 \\ 2 & 3 & 4 & 0 \\ 3 & 4 & 4 & 0 \\ 4 & 5 & 4 & 0 \\ 5 & 6 & 4 & 0 \\ 6 & 7 & 4 & 0 \\ 7 & 8 & 4 & 0 \\ 8 & 9 & 4 & 0 \\ 9 & 1 & 4 & 0 \\ 1 & 2 & 4 & 0 \\ 2 & 3 & 4 & 0 \\ 3 & 4 & 4 & 0 \\ 4 & 5 & 4 & 0 \\ 5 & 6 & 4 & 0 \\ 6 & 7 & 4 & 0 \\ 7 & 8 & 4 & 0\end{array}$




\begin{tabular}{|c|c|c|c|c|c|c|c|c|c|c|c|}
\hline IRP & 2393 & \multicolumn{2}{|l|}{11.48} & 8 & 9 & & 4 & \multicolumn{4}{|l|}{0} \\
\hline IRP & 2452 & \multicolumn{2}{|l|}{10.91} & 9 & 1 & & 4 & \multicolumn{4}{|l|}{0} \\
\hline IRP & 2483 & 10.33 & & 1 & 2 & & 4 & \multicolumn{4}{|l|}{0} \\
\hline $\mathrm{DEC}$ & \multicolumn{3}{|l|}{2531} & 2 & 3 & & 4 & \multicolumn{4}{|l|}{0} \\
\hline IRP & \multicolumn{3}{|l|}{2539} & 3 & 4 & & 4 & \multicolumn{4}{|l|}{0} \\
\hline IRP & 2548 & 8.75 & & 4 & 5 & & 4 & \multicolumn{4}{|l|}{0} \\
\hline IRP & 2627 & 10.71 & & 5 & 6 & & 4 & \multicolumn{4}{|l|}{0} \\
\hline $\mathrm{DEC}$ & 2688 & & & 6 & 7 & & 4 & \multicolumn{4}{|l|}{0} \\
\hline IRP & 2743 & 10.71 & & 7 & $\varepsilon$ & & 4 & \multicolumn{4}{|l|}{0} \\
\hline IRP & 2817 & 10.39 & & 8 & 9 & & 4 & \multicolumn{4}{|l|}{0} \\
\hline $\mathrm{DEC}$ & 2822 & & & 9 & 1 & & 4 & 0 & & & \\
\hline IRP & 2853 & 9.84 & & 1 & 2 & & 4 & 0 & & & \\
\hline IRP & 2879 & 8.75 & & 2 & 3 & & 4 & 0 & & & \\
\hline BUP & & & & & & & & & & & \\
\hline BAS & 46 Rods & & & & & & & & & & \\
\hline RDA & $3.89 \mathrm{Kg}$ & U / Rod & & & & & & & & & \\
\hline MOV & $\begin{array}{lll}3 & 3 & 0\end{array}$ & .1789 & & & & & & & & & \\
\hline DEC & 1 & & & 3 & 4 & & 5 & 2 & & & \\
\hline $\mathrm{DEC}$ & 5 & & & 4 & 5 & & 5 & 0 & & & \\
\hline $\mathrm{DEC}$ & 14.1 & & & 5 & 1 & & 5 & 0 & & & \\
\hline $\mathrm{DEC}$ & 18.1 & & & 1 & 2 & & 5 & 0 & & & \\
\hline $\mathrm{DEC}$ & 28.1 & & & 2 & 3 & & 5 & 0 & & & \\
\hline $\mathrm{DEC}$ & 48.1 & & & 3 & 4 & & 5 & 0 & & & \\
\hline $\mathrm{DEC}$ & 118.1 & & & 4 & 5 & & 5 & 0 & & & \\
\hline HED & 1 June & 1996 & & & & & & & & & \\
\hline HED & 2 June & 2000 & & & & & & & & & \\
\hline HED & 3 June & 2010 & & & & & & & & & \\
\hline HED & June & 2030 & & & & & & & & & \\
\hline HED & June & 2100 & & & & & & & & & \\
\hline OPTL & $\begin{array}{llll}8 & 8 & 8 & 8\end{array}$ & $\begin{array}{llll}7 & 8 & 7 & 8\end{array}$ & 38 & 8 & 8 & 8 & 8 & 888 & $\begin{array}{llll}8 & 8 & 8 & 8\end{array}$ & 88 & \\
\hline OPTA & $\begin{array}{llll}8 & 8 & 8 & 8\end{array}$ & $\begin{array}{lllll}3 & 7 & 8 & 7 & 8\end{array}$ & 78 & 8 & 8 & 8 & 8 & 888 & $\begin{array}{llll}8 & 8 & 8 & 8\end{array}$ & 88 & \\
\hline OPTE & $\begin{array}{llll}8 & 8 & 8 & 8\end{array}$ & $\begin{array}{llll}7 & 8 & 7 & 8\end{array}$ & 78 & 8 & $8 \varepsilon$ & 8 & 8 & 888 & $\begin{array}{llll}8 & 8 & 8 & 8\end{array}$ & 88 & \\
\hline CUT & $51 .-5$ & $71 .-10$ & -1 & & & & & & & & \\
\hline OUT' & 5110 & 0 & & & & & & & & & \\
\hline STP & 4 & & & & & & & & & & \\
\hline 2 & 922340 & 227 & 92235 & & 250 & 00 & . & 922380 & 974773 . & 00.0 & \\
\hline 4 & 80000 & 134470 . & 6000 & & 20 & 4 & & 70000 & 26.1 & 90000 & 5.7 \\
\hline 4 & 170000 & 5.7 & 26000 & & 51 & 1 & & 470000 & 1.1 & 200000 & 36.3 \\
\hline 4 & 130000 & 36.3 & 14000 & & 36. & & & 280000 & 28.4 & 00.0 & \\
\hline 0 & & & & & & & & & & & \\
\hline & & & & & & & & & & & \\
\hline
\end{tabular}


$-1$

TIT

BAS

BWR Cooper-Assembly CZ348-ATM-105/108 E=2.50 27.48 GWD/MTU

$\begin{array}{lllllllllllll}\text { LIB } & 0 & 1 & 2 & 3 & 251 & 252 & 253 & 9 & 3 & 0 & 1 & 4\end{array}$

PHO $\quad 101 \quad 102 \quad 103 \quad 10$

IIP $\quad 0 \quad 0 \quad 0$

$\begin{array}{lllllll}\text { INP } & 1 & 1 & -1 & -1 & 1 & 1\end{array}$

RDA Burnup to $27,480 \mathrm{MWD} / \mathrm{MTU}$

BUP

IRP

IRP

IRP

IRP

DEC

IRP

IRP

DEC

IRP

DEC

IRP

DEC

IRP

BUP

BAS

RDA

MOV

DEC

$D E C$

$D E C$

DEC

DEC

DEC

DEC

HED

HED

HED

HED

HED

OPTL

$\begin{array}{rccccc}200 & 16.25 & 1 & 2 & 4 & 2 \\ 400 & 16.25 & 2 & 3 & 4 & 0 \\ 600 & 16.25 & 3 & 4 & 4 & 0 \\ 806 & 16.25 & 4 & 5 & 4 & 0 \\ 866 & & 5 & 6 & 4 & 0 \\ 1020 & 19.44 & 6 & 7 & 4 & 0 \\ 1171 & 19.44 & 7 & 8 & 4 & 0 \\ 1202 & & 8 & 9 & 4 & 0 \\ 1366 & 18.78 & 9 & 1 & 4 & 0 \\ 2166 & & 1 & 2 & 4 & 0 \\ 2482 & 8.42 & 2 & 3 & 4 & 0 \\ 2531 & & 3 & 4 & 4 & 0 \\ 2878 & 7.81 & 4 & 5 & 4 & 0\end{array}$

OPTA

OPTF

CUT

OUT

STP

2

4

4

4

0

49 Rods

$3.89 \mathrm{KgU} / \mathrm{Rod}$

$5 \quad 5 \quad 0 \quad .1906$

1 .

5.

14.1

18.1

28.1

48.1

118.1

1 June 1996

2 June 2000

3 June 2010

4 June 2030

5 June 2100

$\begin{array}{llllllllllllllllllllllll}8 & 8 & 8 & 8 & 7 & 8 & 7 & 8 & 7 & 8 & 8 & 8 & 8 & 8 & 8 & 8 & 8 & 8 & 8 & 8 & 8 & 8 & 8 & 8\end{array}$

$\begin{array}{llllllllllllllllllllllll}8 & 8 & 8 & 8 & 7 & 8 & 7 & 8 & 7 & 8 & 8 & 8 & 8 & 8 & 8 & 8 & 8 & 8 & 8 & 8 & 8 & 8 & 8 & 8\end{array}$

$\begin{array}{llllllllllllllllllllllll}8 & 8 & 8 & 8 & 7 & 8 & 7 & 8 & 7 & 8 & 8 & 8 & 8 & 8 & 8 & 8 & 8 & 8 & 8 & 8 & 8 & 8 & 8 & 8\end{array}$

5 1. $-5 \quad 5 \quad 7 \quad 1 .-10 \quad-1$

$\begin{array}{llll}5 & 1 & 0 & 0\end{array}$

4

$922340227 . \quad 92235025000.922380 \quad 974773.000$

$80000134470.6000020 .4 \quad 7000026.1 \quad 90000 \quad 5.7$

$1700005.7 \quad 26000051.1 \quad 4700001.1$

$13000036.3 \quad 14000036.3$

28000028.4

$200000 \quad 36: 3$

0.0

END 
$-1$

TIT

BAS

LIP

LIB

$\mathrm{PHO}$

INP

MOV

PWR - Point Beach Ave. Assembly E=3.192

$32,937 \mathrm{MWD} / \mathrm{MTU}$

MTU

RDA BURNUP TO $32,937 \mathrm{MWD} / \mathrm{MTU}$

BUP

IRP

IRP

DEC

IRP

IRP

IRP

DEC

IRP

IRP

IRP

DEC

IRP

DEC

IRP

BUP

BAS

RDA

MOV

DEC

DEC

$D E C$

DEC

DEC

DEC

$D E C$

HED

HED

HED

HED

HED

OPTL

OPTA

OPTF

CUT

OUT

STP

2

4

0

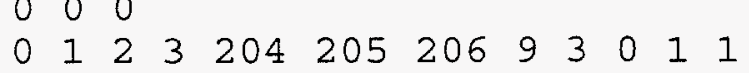

$\begin{array}{llll}101 & 102 & 103 & 10\end{array}$

$\begin{array}{llllll}1 & 1 & -1 & -1 & 1 & 1\end{array}$

$\begin{array}{llll}1 & 1 & 0 & 2.4932\end{array}$

$\begin{array}{rrrrrr}158 & 20.541 & 1 & 2 & 4 & 2 \\ 316 & 20.541 & 2 & 3 & 4 & 0 \\ 346 & & 3 & 4 & 4 & 0 \\ 453 & 33.071 & 4 & 5 & 4 & 0 \\ 560 & 33.071 & 5 & 6 & 4 & 0 \\ 667 & 33.071 & 6 & 7 & 4 & 0 \\ 691 & & 7 & 8 & 4 & 0 \\ 810 & 26.204 & 8 & 9 & 4 & 0 \\ 928 & 26.204 & 9 & 1 & 4 & 0 \\ 1047 & 26.204 & 1 & 2 & 4 & 0 \\ 1104 & & 2 & 3 & 4 & 0 \\ 1465 & 9.482 & 3 & 4 & 4 & 0 \\ 1497 & & 4 & 5 & 4 & 0 \\ 1782 & 10.804 & 5 & 6 & 4 & 0\end{array}$

1 Assembly (401.094 $\mathrm{KgU})$

$2.241 \mathrm{KgU} / \mathrm{Rod}$

$\begin{array}{llll}6 & 6 & 0 & .401094\end{array}$

$\begin{array}{lllll}1 . & 6 & 7 & 5 & 2 \\ 5 . & 7 & 8 & 5 & 0 \\ 14.7 & 8 & 1 & 5 & 0 \\ 18.7 & 1 & 2 & 5 & 0 \\ 28.7 & 2 & 3 & 5 & 0 \\ 48.7 & 3 & 4 & 5 & 0 \\ 118.7 & 4 & 5 & 5 & 0\end{array}$

END 
Appendix B

Decay Heat and Source Terms 
Table B.1. Decay Heat and Source Terms for Year 2000

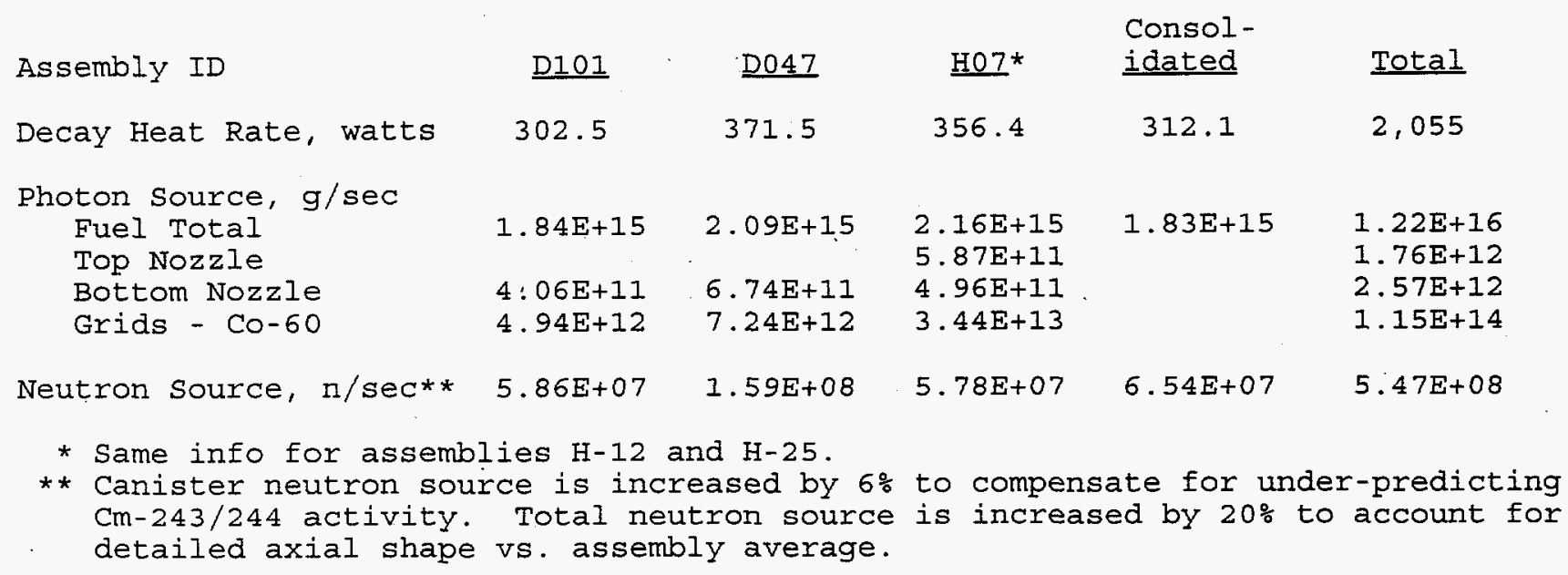

Table B.2. Decay Heat and Source Terms for Year 2010

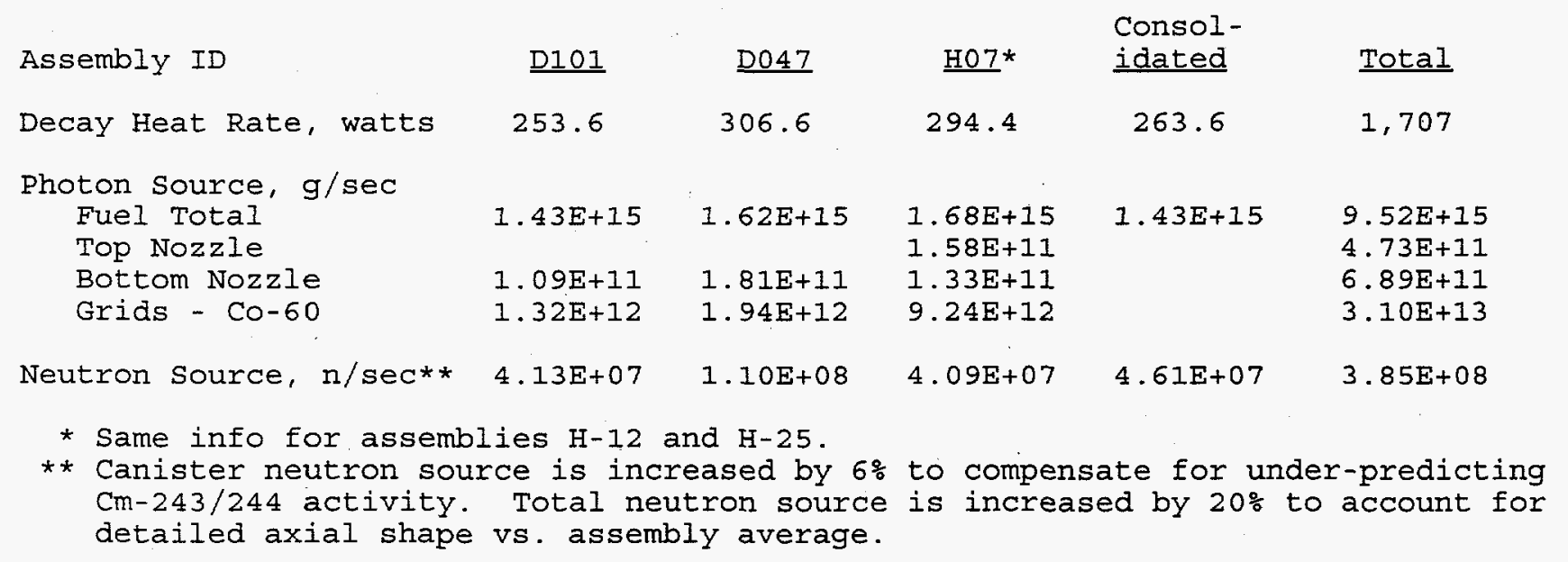


Table B.3. Decay Heat and Source Terms for Year 2030

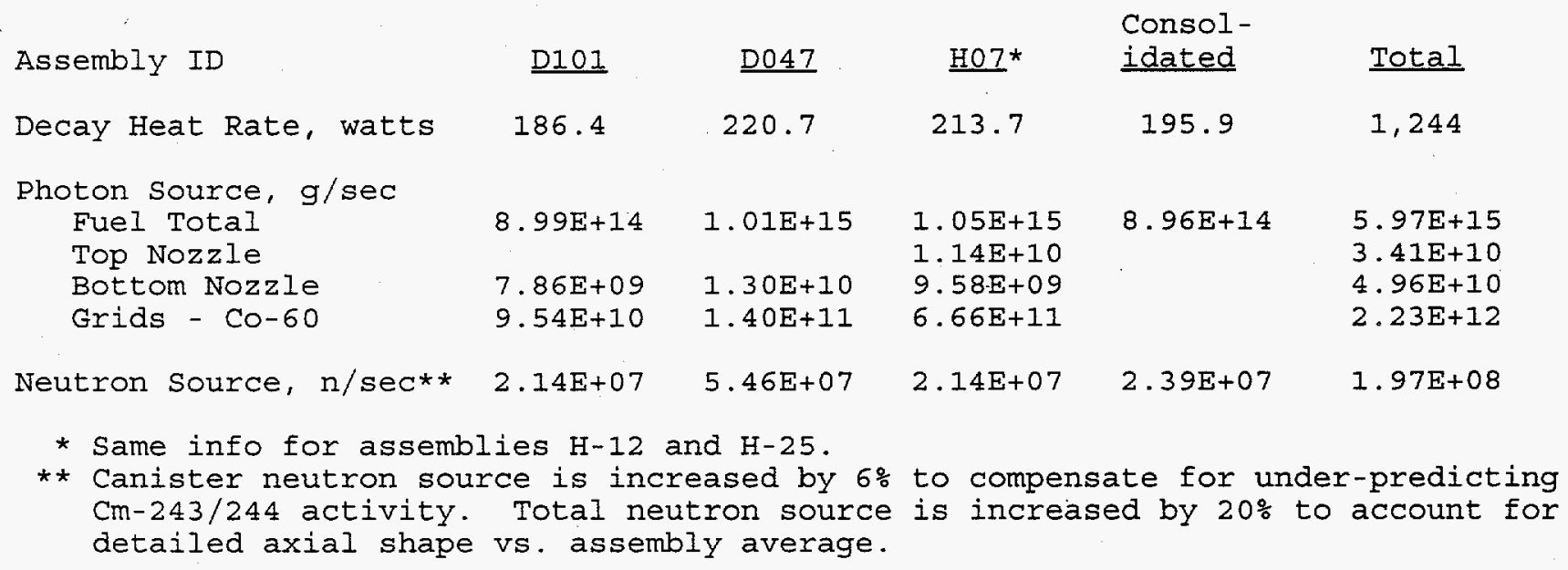

Table B.4. Decay Heat and Source Terms for Year 2100

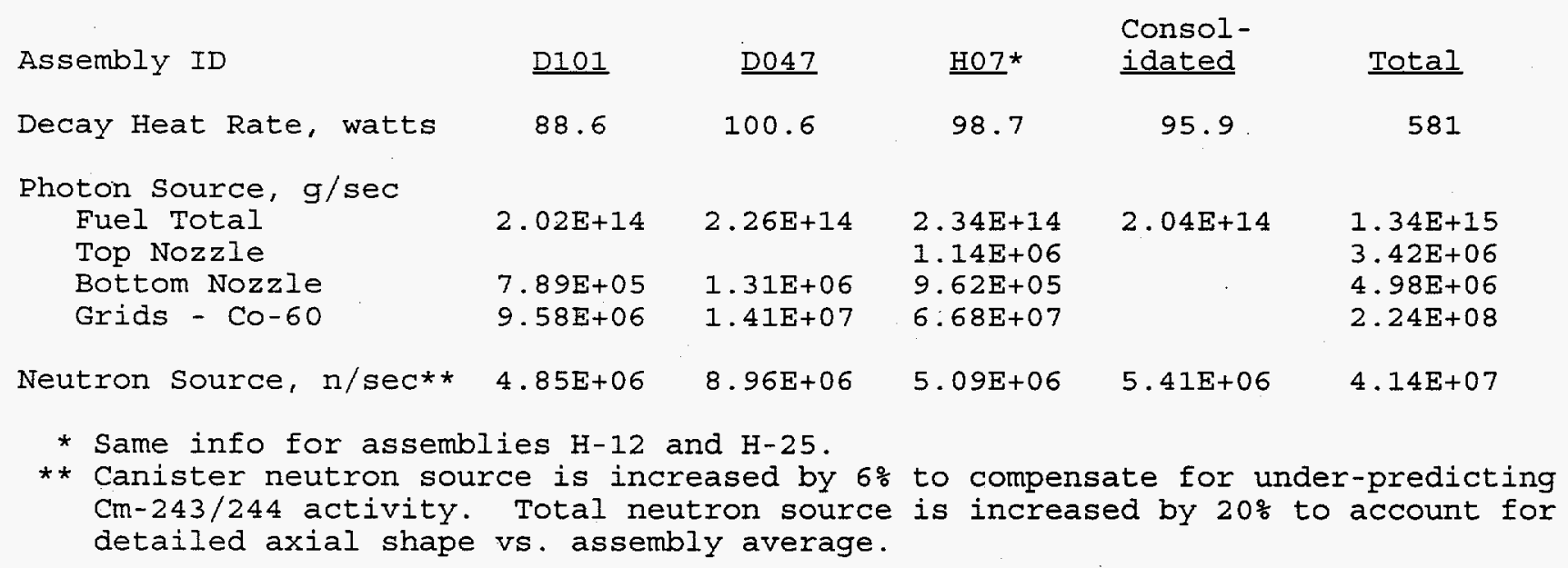


Table B.5. Calculated Photon Spectra for Assembly D047, photons/sec

\begin{tabular}{|c|c|c|c|c|c|}
\hline $\mathrm{E}, \mathrm{MeV}$ & June 1996 & Year 2000 & Year 2010 & Year 2030 & Year 2100 \\
\hline 0.010 & $6.00 E+14$ & $5.43 E+14$ & $4.28 E+14$ & $2.71 E+14$ & $6.48 \mathrm{E}+13$ \\
\hline 0.025 & $1.21 E+14$ & $1.08 E+14$ & $8.39 E+13$ & $5.24 E+13$ & $1.11 E+13$ \\
\hline 0.0375 & $1.58 \mathrm{E}+14$ & $1.39 \mathrm{E}+14$ & $1.05 E+14$ & $6.34 \mathrm{E}+13$ & $1.22 E+13$ \\
\hline 0.0575 & 1. $23 E+14$ & 1. $13 \mathrm{E}+14$ & $9.39 \mathrm{E}+13$ & $6.71 \mathrm{E}+13$ & $2.88 E+13$ \\
\hline 0.085 & $6.84 E+13$ & $6.03 E+13$ & $4.58 E+13$ & $2.81 E+13$ & $5.63 E+12$ \\
\hline 0.125 & $6.62 E+13$ & $5.49 E+13$ & $3.67 \mathrm{E}+13$ & $1.95 \mathrm{E}+13$ & 3. $61 E+12$ \\
\hline 0.225 & $5.70 E+13$ & $5.07 \mathrm{E}+13$ & $3.86 \mathrm{E}+13$ & $2.34 E+13$ & $4.49 E+12$ \\
\hline 0.375 & $2.43 E+13$ & $2.10 E+13$ & $1.59 \mathrm{E}+13$ & $9.75 \mathrm{E}+12$ & $1.86 \mathrm{E}+12$ \\
\hline 0.575 & $1.06 E+15$ & $9.49 \mathrm{E}+14$ & $7.46 \mathrm{E}+14$ & $4.69 \mathrm{E}+14$ & $9.30 E+13$ \\
\hline 0.85 & $4.68 E+13$ & $2.72 \mathrm{E}+13$ & I. $15 \mathrm{E}+13$ & $3.32 \mathrm{E}+12$ & $3.01 E+11$ \\
\hline 1.25 & $3.25 E+13$ & $2.33 E+13$ & $1.06 \mathrm{E}+13$ & $2.47 \mathrm{E}+12$ & $1.04 E+11$ \\
\hline 1.75 & $9.98 E+11$ & $7.38 \mathrm{E}+11$ & $3.57 \mathrm{E}+11$ & $9.83 E+10$ & $7.71 E+09$ \\
\hline 2.25 & $6.88 E+08$ & $7.23 E+07$ & $2.69 E+07$ & $1.40 \mathrm{E}+07$ & $2.17 E+06$ \\
\hline 2.75 & $2.89 E+08$ & $2.23 E+08$ & $1.99 \mathrm{E}+08$ & $1.61 \mathrm{E}+08$ & $7.98 E+07$ \\
\hline 3.5 & $2.62 E+07$ & $1.56 E+07$ & $1.04 E+07$ & $5.02 E+06$ & $6.76 E+05$ \\
\hline 5.0 & $7.46 \mathrm{E}+06$ & $6.43 E+06$ & $4.43 E+06$ & $2.14 E+06$ & $2.88 E+05$ \\
\hline 7.0 & $8.61 \mathrm{E}+05$ & $7.41 \mathrm{E}+05$ & $5.11 E+05$ & $2.47 \mathrm{E}+05$ & $3.29 E+04$ \\
\hline 9.5 & $9.89 E+04$ & $8.51 E+04$ & $5.87 \mathrm{E}+04$ & $2.84 E+04$ & $3.77 E+03$ \\
\hline Total & $2.36 \mathrm{E}+15$ & $2.09 E+15$ & $1.62 E+15$ & $1.01 E+15$ & $2.26 E+14$ \\
\hline
\end{tabular}


Appendix C

Radioactivity and Mass Inventories 
Table C.1. LWR Spent Fuel Radioactivity as of June 1996, Ci/Canister

\begin{tabular}{|c|c|c|c|c|c|c|}
\hline & \multicolumn{2}{|c|}{ Calvert Cliffs } & \multicolumn{3}{|c|}{ Point Beach } & \multirow{2}{*}{ Consolidated } \\
\hline Nuclide & D101 & D047 & $\mathrm{H}-07$ & $\mathrm{H}-12$ & $\mathrm{H}-25$ & \\
\hline$A m-241$ & $8.80 \mathrm{E}+02$ & $8.72 \mathrm{E}+02$ & $9.20 \mathrm{E}+02$ & $9.20 \mathrm{E}+02$ & $9.20 \mathrm{E}+02$ & $9.44 \mathrm{E}+02$ \\
\hline $\mathrm{Am}-242$ & $3.65 \mathrm{E}+00$ & $4.56 E+00$ & $6.37 E+00$ & $6.37 E+00$ & $6.37 E+00$ & $1.07 \mathrm{E}+01$ \\
\hline $\mathrm{Am}-242 \mathrm{~m}$ & $3.66 \mathrm{E}+00$ & $4.59 \mathrm{E}+00$ & $6.40 \mathrm{E}+00$ & $6.40 \mathrm{E}+00$ & $6.40 \mathrm{E}+00$ & $1.07 \mathrm{E}+01$ \\
\hline Am-243 & $6.86 \mathrm{E}+00$ & $1.28 \mathrm{E}+01$ & $6.82 E+00$ & $6.82 \mathrm{E}+00$ & $6.82 \mathrm{E}+00$ & $7.31 \mathrm{E}+00$ \\
\hline $\mathrm{Ba}-137 \mathrm{~m}$ & $2.33 E+04$ & $2.67 E+04$ & $2.70 E+04$ & $2.70 \mathrm{E}+04$ & $2.70 \mathrm{E}+04$ & $2.32 E+04$ \\
\hline $\mathrm{Cd}-113 \mathrm{~m}$ & $9.74 E+00$ & $1.31 \mathrm{E}+01$ & $1.05 \mathrm{E}+01$ & $1.05 \mathrm{E}+01$ & $1.05 \mathrm{E}+01$ & $9.01 E+00$ \\
\hline $\mathrm{Cm}-242$ & $3.02 E+00$ & $3.77 \mathrm{E}+00$ & $5.27 \mathrm{E}+00$ & $5.27 \mathrm{E}+00$ & $5.27 \mathrm{E}+00$ & $8.82 \mathrm{E}+00$ \\
\hline $\mathrm{Cm}-243$ & $5.83 \mathrm{E}+00$ & $1.05 \mathrm{E}+01$ & $4.68 \mathrm{E}+00$ & $4.68 \mathrm{E}+00$ & $4.68 \mathrm{E}+00$ & $7.33 \mathrm{E}+00$ \\
\hline $\mathrm{Cm}-244$ & $4.35 E+02$ & 1. $21 \mathrm{E}+03$ & $4.26 \mathrm{E}+02$ & $4.26 \mathrm{E}+02$ & 4. $26 E+02$ & $4.84 E+02$ \\
\hline $\mathrm{Co}-60$ & $1.13 E+02$ & $1.66 \mathrm{E}+02$ & $7.88 \mathrm{E}+02$ & $7.88 \mathrm{E}+02$ & $7.88 \mathrm{E}+02$ & $1.23 \mathrm{E}-02$ \\
\hline $\mathrm{Cs}-134$ & $2.44 E+02$ & $4.91 \mathrm{E}+02$ & $3.07 \mathrm{E}+02$ & $3.07 \mathrm{E}+02$ & $3.07 E+0.2$ & $2.56 \mathrm{E}+02$ \\
\hline $\mathrm{Cs}-137$ & $2.47 \mathrm{E}+04$ & $2.82 \mathrm{E}+04$ & $2.85 E+04$ & $2.85 \mathrm{E}+04$ & $2.85 E+04$ & $2.46 \mathrm{E}+04$ \\
\hline$E u-154$ & $1.04 \mathrm{E}+03$ & $1.55 \mathrm{E}+03$ & $1.20 \mathrm{E}+03$ & $1.20 \mathrm{E}+03$ & $1.20 \mathrm{E}+03$ & $1.09 \mathrm{E}+03$ \\
\hline$E u-155$ & $2.53 \mathrm{E}+02$ & $4.02 E+02$ & $2.97 \mathrm{E}+02$ & $2.97 \mathrm{E}+02$ & $2.97 E+02$ & $2.74 E+02$ \\
\hline $\mathrm{H}-3$ & $7.81 E+01$ & $9.28 \mathrm{E}+01$ & $8.86 E+01$ & $8.86 \mathrm{E}+01$ & $8.86 \mathrm{E}+01$ & $7.48 \mathrm{E}+01$ \\
\hline$I-129$ & $1.12 \mathrm{E}-02$ & 1. $26 \mathrm{E}-02$ & $1.26 E-02$ & $1.26 \mathrm{E}-02$ & $1.26 \mathrm{E}-02$ & $1.15 \mathrm{E}-02$ \\
\hline $\mathrm{Kr}-85$ & $1.09 \mathrm{E}+03$ & $1.22 E+03$ & $1.31 E+03$ & $1.31 E+03$ & $1.31 E+03$ & $1.02 \mathrm{E}+03$ \\
\hline $\mathrm{Np}-237$ & $1.07 \mathrm{E}-01$ & $1.30 \mathrm{E}-01$ & $1.34 \mathrm{E}-01$ & $1.34 \mathrm{E}-01$ & i. $34 \mathrm{E}-0 \mathrm{I}$ & $1.07 \mathrm{E}-01$ \\
\hline$N p-239$ & $6.86 \mathrm{E}+00$ & $1.28 E+01$ & $6.82 E+00$ & $6.82 \mathrm{E}+00$ & $6.82 E+00$ & $7.31 \mathrm{E}+00$ \\
\hline$P m-147$ & $6.72 \mathrm{E}+02$ & $7.66 \mathrm{E}+02$ & $8.17 E+02$ & $8.17 E+02$ & $8.17 \mathrm{E}+02$ & $6.77 \mathrm{E}+02$ \\
\hline Pu-238 & $8.34 \mathrm{E}+02$ & 1. $36 E+03$ & $1.04 \mathrm{E}+03$ & $1.04 \mathrm{E}+03$ & $1.04 \mathrm{E}+03$ & $1.07 E+03$ \\
\hline Pu-239 & $1.16 E+02$ & $9.78 \mathrm{E}+01$ & $1.25 E+02$ & $1.25 \mathrm{E}+02$ & $1.25 \mathrm{E}+02$ & $1.23 E+02$ \\
\hline$P u-240$ & $1.96 E+02$ & 1. $92 E+02$ & $2.12 E+02$ & $2.12 \mathrm{E}+02$ & $2.12 \mathrm{E}+02$ & $2.06 \mathrm{E}+02$ \\
\hline$P u-241$ & $2.22 \mathrm{E}+04$ & $2.44 \mathrm{E}+04$ & $2.38 E+04$ & $2.38 \mathrm{E}+04$ & 2. $38 \mathrm{E}+04$ & $2.37 \mathrm{E}+04$ \\
\hline $\mathrm{Pu}-242$ & $6.76 \mathrm{E}-01$ & $9.39 E-01$ & $6.84 \mathrm{E}-01$ & $6.84 \mathrm{E}-01$ & $6.84 \mathrm{E}-01$ & $6.99 \mathrm{E}-01$ \\
\hline $\mathrm{Rh}-106$ & $3.40 \mathrm{E}+00$ & $8.43 E+00$ & $4.36 \mathrm{E}+00$ & $4.36 \mathrm{E}+00$ & $4.36 \mathrm{E}+00$ & $4.45 \mathrm{E}+00$ \\
\hline $\mathrm{Ru}-106$ & $3.40 \mathrm{E}+00$ & $8.42 \mathrm{E}+00$ & $4.34 E+00$ & $4.34 \mathrm{E}+00$ & $4.34 \mathrm{E}+00$ & $4.44 \mathrm{E}+00$ \\
\hline$S b-125$ & $9.35 E+01$ & $1.30 \mathrm{E}+02$ & $1.05 E+02$ & $1.05 E+02$ & $1.05 \mathrm{E}+02$ & $7.79 \mathrm{E}+01$ \\
\hline Sm-15I & $1.10 \mathrm{E}+02$ & $1.06 \mathrm{E}+02$ & $1.23 E+02$ & 1. $23 \mathrm{E}+02$ & $1.23 E+02$ & $1.14 \mathrm{E}+02$ \\
\hline$S r-90$ & $1.64 \mathrm{E}+04$ & 1. $78 \mathrm{E}+04$ & $1.98 \mathrm{E}+04$ & $1.98 \mathrm{E}+04$ & $1.98 \mathrm{E}+04$ & $1.62 \mathrm{E}+04$ \\
\hline$T C-99$ & $4.51 \mathrm{E}+00$ & $4.86 \mathrm{E}+00$ & $5.21 \mathrm{E}+00$ & $5.21 \mathrm{E}+00$ & $5.21 E+00$ & $4.66 \mathrm{E}+00$ \\
\hline $\mathrm{Te}-125 \mathrm{~m}$ & $2.28 \mathrm{E}+01$ & $3.17 \mathrm{E}+01$ & $2.56 \mathrm{E}+01$ & $2.56 \mathrm{E}+01$ & $2.56 \mathrm{E}+01$ & $1.90 \mathrm{E}+01$ \\
\hline $\mathrm{U}-234$ & $3.57 E-01$ & $3.02 \mathrm{E}-01$ & 4. $72 \mathrm{E}-01$ & $4.72 \mathrm{E}-01$ & $4.72 \mathrm{E}-01$ & $4.04 \mathrm{E}-01$ \\
\hline $\mathrm{U}-235$ & $5.34 \mathrm{E}-03$ & $2.88 \mathrm{E}-03$ & $6.91 \mathrm{E}-03$ & $6.91 E-03$ & $6.91 \mathrm{E}-03$ & $5.38 \mathrm{E}-03$ \\
\hline $\mathrm{U}-236$ & $8.15 \mathrm{E}-02$ & $7.95 \mathrm{E}-02$ & $1.07 \mathrm{E}-01$ & $1.07 \mathrm{E}-01$ & $1.07 \mathrm{E}-01$ & $8.28 \mathrm{E}-02$ \\
\hline $\mathrm{U}-237$ & $5.44 \mathrm{E}-01$ & $5.99 \mathrm{E}-01$ & $5.83 E-01$ & $5.83 \mathrm{E}-01$ & $5.83 E-0 I$ & $5.82 \mathrm{E}-01$ \\
\hline $\mathrm{U}-238$ & $1.19 \mathrm{E}-01$ & $9.71 \mathrm{E}-02$ & $1.27 \mathrm{E}-01$ & $1.27 \mathrm{E}-01$ & $1.27 \mathrm{E}-01$ & $1.30 \mathrm{E}-01$ \\
\hline$Y-90$ & $1.64 \mathrm{E}+04$ & $1.78 \mathrm{E}+04$ & $1.98 \mathrm{E}+04$ & $1.98 \mathrm{E}+04$ & $1.98 \mathrm{E}+04$ & $1.62 \mathrm{E}+04$ \\
\hline Total & $1.09 \mathrm{E}+05$ & $1.24 \mathrm{E}+05$ & $1.27 E+05$ & $1.27 \mathrm{E}+05$ & $1.27 \mathrm{E}+05$ & $1.10 \mathrm{E}+05$ \\
\hline
\end{tabular}


Table C.2. LWR Spent Fuel Radioactivity as of Year 2000, Ci/Canister

\begin{tabular}{|c|c|c|c|c|c|c|}
\hline & \multicolumn{2}{|c|}{ Calvert Cliffs } & \multicolumn{3}{|c|}{ Point Beach } & \multirow{2}{*}{ Consolidated } \\
\hline Nuclide & D101 & D047 & $\mathrm{H}-07$ & $\mathrm{H}-12$ & $\mathrm{H}-25$ & \\
\hline$A m-241$ & $1.00 \mathrm{E}+03$ & $1.01 E+03$ & $1.05 E+03$ & $1.05 \mathrm{E}+03$ & $1.05 E+03$ & $1.08 E+03$ \\
\hline $\mathrm{Am}-242$ & $3.58 E+00$ & $4.48 \mathrm{E}+00$ & $6.25 E+00$ & $6.25 \mathrm{E}+00$ & $6.25 E+00$ & $1.05 \mathrm{E}+01$ \\
\hline $\mathrm{Am}-242 \mathrm{~m}$ & $3.60 \mathrm{E}+00$ & $4.50 \mathrm{E}+00$ & $6.28 \mathrm{E}+00$ & $6.28 \mathrm{E}+00$ & $6.28 \mathrm{E}+00$ & $1.05 \mathrm{E}+01$ \\
\hline Am-243 & $6.85 \mathrm{E}+00$ & $1.28 \mathrm{E}+01$ & $6.82 E+00$ & $6.82 \mathrm{E}+00$ & $6.82 \mathrm{E}+00$ & $7.30 \mathrm{E}+00$ \\
\hline $\mathrm{Ba}-137 \mathrm{~m}$ & $2.13 E+04$ & $2.43 E+04$ & $2.46 \mathrm{E}+04$ & $2.46 \mathrm{E}+04$ & $2.46 \mathrm{E}+04$ & $2.12 \mathrm{E}+04$ \\
\hline $\mathrm{Cd}-113 \mathrm{~m}$ & $8.05 \mathrm{E}+00$ & $1.08 \mathrm{E}+01$ & $8.65 \mathrm{E}+00$ & $8.65 E+00$ & $8.65 E+00$ & $7.45 \mathrm{E}+00$ \\
\hline $\mathrm{Cm}-242$ & $2.97 \mathrm{E}+00$ & $3.71 \mathrm{E}+00$ & $5.18 \mathrm{E}+00$ & $5.18 \mathrm{E}+00$ & $5.18 \mathrm{E}+00$ & $8.68 \mathrm{E}+00$ \\
\hline $\mathrm{Cm}-243$ & $5.29 \mathrm{E}+00$ & $9.56 \mathrm{E}+00$ & $4.24 E+00$ & $4.24 \mathrm{E}+00$ & $4.24 \mathrm{E}+00$ & $6.65 \mathrm{E}+00$ \\
\hline $\mathrm{Cm}-244$ & $3.73 \mathrm{E}+02$ & $1.04 \mathrm{E}+03$ & $3.65 \mathrm{E}+02$ & $3.65 \mathrm{E}+02$ & $3.65 \mathrm{E}+02$ & $4.15 \mathrm{E}+02$ \\
\hline Co-60 & $6.67 \mathrm{E}+01$ & $9.79 E+01$ & $4.65 \mathrm{E}+02$ & $4.65 \mathrm{E}+02$ & $4.65 \mathrm{E}+02$ & $7.29 \mathrm{E}-03$ \\
\hline $\mathrm{Cs}-134$ & $6.36 E+01$ & $1.28 \mathrm{E}+02$ & $7.99 E+01$ & $7.99 \mathrm{E}+01$ & $7.99 \mathrm{E}+01$ & $6.68 \mathrm{E}+01$ \\
\hline $\mathrm{Cs}-137$ & $2.25 \mathrm{E}+04$ & $2.57 \mathrm{E}+04$ & $2.60 \mathrm{E}+04$ & $2.60 \mathrm{E}+04$ & $2.60 \mathrm{E}+04$ & $2.24 \mathrm{E}+04$ \\
\hline Eu-154 & $7.55 \mathrm{E}+02$ & $1.12 \mathrm{E}+03$ & $8.71 E+02$ & $8.71 \mathrm{E}+02$ & $8.71 E+02$ & $7.87 \mathrm{E}+02$ \\
\hline $\mathrm{Eu}-155$ & $1.45 \mathrm{E}+02$ & $2.30 E+02$ & $1.70 E+02$ & $1.70 \mathrm{E}+02$ & $1.70 \mathrm{E}+02$ & $1.57 \mathrm{E}+02$ \\
\hline $\mathrm{H}-3$ & $8.12 \mathrm{E}-06$ & $2.44 E-05$ & $7.08 \mathrm{E}+01$ & $7.08 \mathrm{E}+01$ & $7.08 \mathrm{E}+01$ & $8.38 E-06$ \\
\hline$I-129$ & $1.12 \mathrm{E}-02$ & $1.26 \mathrm{E}-02$ & $1.26 \mathrm{E}-02$ & $1.26 \mathrm{E}-02$ & $1.26 \mathrm{E}-02$ & $1.15 \mathrm{E}-02$ \\
\hline $\mathrm{Kr}-85$ & $8.44 \mathrm{E}+02$ & $9.45 E+02$ & $1.01 \mathrm{E}+03$ & $1.01 \mathrm{E}+03$ & $1.01 \mathrm{E}+03$ & $7.90 \mathrm{E}+02$ \\
\hline$N p-237$ & 1. $08 \mathrm{E}-01$ & 1. $31 E-01$ & $1.35 \mathrm{E}-01$ & $1.35 \mathrm{E}-01$ & $1.35 \mathrm{E}-01$ & $1.08 \mathrm{E}-01$ \\
\hline $\mathrm{Np}-239$ & $6.85 E+00$ & $1.28 \mathrm{E}+01$ & $6.82 \mathrm{E}+00$ & $6.82 \mathrm{E}+00$ & $6.82 \mathrm{E}+00$ & $7.30 \mathrm{E}+00$ \\
\hline$P m-147$ & $2.34 \mathrm{E}+02$ & $2.66 \mathrm{E}+02$ & $2.84 \mathrm{E}+02$ & $2.84 \mathrm{E}+02$ & $2.84 \mathrm{E}+02$ & $2.35 \mathrm{E}+02$ \\
\hline $\mathrm{Pu}-238$ & $8.08 \mathrm{E}+02$ & $1.31 \mathrm{E}+03$ & $1.01 \mathrm{E}+03$ & $1.01 \mathrm{E}+03$ & $1.01 \mathrm{E}+03$ & $1.03 E+03$ \\
\hline $\mathrm{Pu}-239$ & $1.16 \mathrm{E}+02$ & $9.78 \mathrm{E}+01$ & $1.25 \mathrm{E}+02$ & $1.25 \mathrm{E}+02$ & $1.25 \mathrm{E}+02$ & $1.23 \mathrm{E}+02$ \\
\hline Pu-240 & $1.96 \mathrm{E}+02$ & $1.92 \mathrm{E}+02$ & $2.12 \mathrm{E}+02$ & $2.12 \mathrm{E}+02$ & $2.12 \mathrm{E}+02$ & $2.06 \mathrm{E}+02$ \\
\hline Pu-241 & $1.83 \mathrm{E}+04$ & $2.02 E+04$ & $1.96 \mathrm{E}+04$ & $1.96 \mathrm{E}+04$ & $1.96 \mathrm{E}+04$ & $1.96 \mathrm{E}+04$ \\
\hline Pu-242 & $6.76 \mathrm{E}-01$ & $9.39 \mathrm{E}-01$ & $6.84 \mathrm{E}-01$ & $6.84 \mathrm{E}-01$ & $6.84 \mathrm{E}-01$ & $6.99 \mathrm{E}-01$ \\
\hline $\mathrm{Rh}-106$ & $2.17 \mathrm{E}-01$ & $5.39 E-01$ & $2.78 \mathrm{E}-01$ & $2.78 \mathrm{E}-01$ & $2.78 \mathrm{E}-01$ & $2.84 \mathrm{E}-01$ \\
\hline $\mathrm{Ru}-106$ & $2.17 \mathrm{E}-01$ & $5.39 \mathrm{E}-01$ & $2.78 \mathrm{E}-01$ & $2.78 \mathrm{E}-0 \mathrm{I}$ & $2.78 \mathrm{E}-01$ & $2.84 \mathrm{E}-01$ \\
\hline$S b-125$ & $3.44 \mathrm{E}+01$ & $4.77 E+01$ & $3.85 \mathrm{E}+01$ & $3.85 E+01$ & $3.85 \mathrm{E}+01$ & $2.86 \mathrm{E}+01$ \\
\hline $\mathrm{Sm}-151$ & $1.07 E+02$ & $1.03 E+02$ & $1.19 \mathrm{E}+02$ & $1.19 \mathrm{E}+02$ & $1.19 \mathrm{E}+02$ & $1.11 \mathrm{E}+02$ \\
\hline$S r-90$ & $1.49 E+04$ & $1.62 \mathrm{E}+04$ & $1.80 \mathrm{E}+04$ & $1.80 \mathrm{E}+04$ & $1.80 \mathrm{E}+04$ & $1.47 \mathrm{E}+04$ \\
\hline TC-99 & $4.51 \mathrm{E}+00$ & $4.86 E+00$ & $5.21 \mathrm{E}+00$ & $5.21 \mathrm{E}+00$ & $5.21 \mathrm{E}+00$ & $4.66 \mathrm{E}+00$ \\
\hline $\mathrm{Te}-125 \mathrm{~m}$ & $8.39 \mathrm{E}+00$ & $1.16 \mathrm{E}+01$ & $9.40 \mathrm{E}+00$ & $9.40 \mathrm{E}+00$ & $9.40 \mathrm{E}+00$ & $6.99 \mathrm{E}+00$ \\
\hline $\mathrm{U}-234$ & $3.66 \mathrm{E}-01$ & $3.17 E-01$ & $4.84 E-01$ & $4.84 \mathrm{E}-01$ & $4.84 \mathrm{E}-01$ & $4.15 \mathrm{E}-01$ \\
\hline $\mathrm{U}-235$ & $5.34 \mathrm{E}-03$ & $2.88 E-03$ & $6.91 \mathrm{E}-03$ & $6.91 \mathrm{E}-03$ & $6.91 \mathrm{E}-03$ & $5.39 \mathrm{E}-03$ \\
\hline $\mathrm{U}-236$ & $8.15 \mathrm{E}-02$ & $7.95 \mathrm{E}-02$ & $1.07 \mathrm{E}-01$ & $1.07 \mathrm{E}-01$ & $1.07 \mathrm{E}-01$ & $8.28 \mathrm{E}-02$ \\
\hline $\mathrm{U}-237$ & $4.48 \mathrm{E}-01$ & $4.94 E-01$ & $4.81 \mathrm{E}-01$ & $4.81 \mathrm{E}-01$ & $4.81 E-01$ & $4.80 \mathrm{E}-01$ \\
\hline $\mathrm{U}-238$ & $1.19 \mathrm{E}-01$ & $9.71 E-02$ & $1.27 \mathrm{E}-01$ & $1.27 \mathrm{E}-01$ & $1.27 \mathrm{E}-01$ & $1.30 \mathrm{E}-01$ \\
\hline$Y-90$ & $1.49 \mathrm{E}+04$ & $1.62 \mathrm{E}+04$ & $1.80 \mathrm{E}+04$ & $1.80 \mathrm{E}+04$ & $1.80 \mathrm{E}+04$ & $1.47 \mathrm{E}+04$ \\
\hline Total & $9.67 \mathrm{E}+04$ & $1.09 \mathrm{E}+05$ & $1.12 \mathrm{E}+05$ & $1.12 \mathrm{E}+05$ & $1.12 \mathrm{E}+05$ & $9.76 \mathrm{E}+04$ \\
\hline
\end{tabular}


Table C.3. LWR Spent Fuel Radioactivity as of Year 2010, Ci/Canister

\begin{tabular}{|c|c|c|c|c|c|c|}
\hline & \multicolumn{2}{|c|}{ Calvert Cliffs } & \multicolumn{3}{|c|}{ Point Beach } & \multirow{2}{*}{ Consolidated } \\
\hline Nuclide & D101 & D047 & $\mathrm{H}-07$ & $\mathrm{H}-12$ & $\mathrm{H}-25$ & \\
\hline Am-241 & $1.22 \mathrm{E}+03$ & $1.25 E+03$ & $1.28 \mathrm{E}+03$ & $1.28 \mathrm{E}+03$ & $1.28 \mathrm{E}+03$ & $1.31 E+03$ \\
\hline Am-242 & $3.42 \mathrm{E}+00$ & $4.28 \mathrm{E}+00$ & $5.97 E+00$ & $5.97 \mathrm{E}+00$ & $5.97 \mathrm{E}+00$ & $1.00 \mathrm{E}+01$ \\
\hline $\mathrm{Am}-242 \mathrm{~m}$ & $3.44 \mathrm{E}+00$ & $4.30 \mathrm{E}+00$ & $6.00 \mathrm{E}+00$ & $6.00 \mathrm{E}+00$ & $6.00 \mathrm{E}+00$ & $1.01 E+01$ \\
\hline $\mathrm{Am}-243$ & $6.85 E+00$ & $1.28 \mathrm{E}+01$ & $6.81 E+00$ & $6.81 E+00$ & $6.81 E+00$ & $7.30 \mathrm{E}+00$ \\
\hline $\mathrm{Ba}-137 \mathrm{~m}$ & $1.69 E+04$ & $1.93 \mathrm{E}+04$ & $1.95 \mathrm{E}+04$ & $1.95 \mathrm{E}+04$ & $1.95 \mathrm{E}+04$ & $1.68 \mathrm{E}+04$ \\
\hline $\mathrm{cd}-113 \mathrm{~m}$ & $5.01 E+00$ & $6.74 \mathrm{E}+00$ & $5.38 \mathrm{E}+00$ & $5.38 \mathrm{E}+00$ & $5.38 \mathrm{E}+00$ & $4.63 E+00$ \\
\hline $\mathrm{Cm}-242$ & $2.83 E+00$ & $3.54 \mathrm{E}+00$ & $4.94 \mathrm{E}+00$ & $4.94 E+00$ & $4.94 \mathrm{E}+00$ & $8.27 E+00$ \\
\hline $\mathrm{Cm}-243$ & $4.15 \mathrm{E}+00$ & $7.50 \mathrm{E}+00$ & $3.33 E+00$ & $3.33 E+00$ & $3.33 E+00$ & $5.22 E+00$ \\
\hline $\mathrm{Cm}-244$ & $2.54 \mathrm{E}+02$ & $7.09 \mathrm{E}+02$ & $2.49 E+02$ & $2.49 \mathrm{E}+02$ & $2.49 \mathrm{E}+02$ & $2.83 E+02$ \\
\hline $\mathrm{Co}-60$ & $1.79 \mathrm{E}+0 \mathrm{I}$ & $2.63 E+01$ & $1.25 \mathrm{E}+02$ & $1.25 \mathrm{E}+02$ & $1.25 \mathrm{E}+02$ & $1.96 \mathrm{E}-03$ \\
\hline $\mathrm{Cs}-134$ & $2.21 E+00$ & $4.43 E+00$ & $2.77 \mathrm{E}+00$ & $2.77 \mathrm{E}+00$ & $2.77 \mathrm{E}+00$ & $2.32 \mathrm{E}+00$ \\
\hline $\mathrm{Cs}-137$ & $1.79 \mathrm{E}+04$ & $2.04 \mathrm{E}+04$ & $2.07 E+04$ & $2.07 E+04$ & $2.07 \mathrm{E}+04$ & $1.78 \mathrm{E}+04$ \\
\hline $\mathrm{Eu}-154$ & $3.37 E+02$ & $5.02 \mathrm{E}+02$ & $3.89 E+02$ & $3.89 \mathrm{E}+02$ & $3.89 E+02$ & $3.51 E+02$ \\
\hline$E u-155$ & $3.58 \mathrm{E}+01$ & $5.69 E+01$ & $4.19 E+01$ & $4.19 \mathrm{E}+01$ & $4.19 E+01$ & $3.88 \mathrm{E}+01$ \\
\hline $\mathrm{H}-3$ & $4.63 E-06$ & $1.39 \mathrm{E}-05$ & $4.04 \mathrm{E}+01$ & $4.04 \mathrm{E}+01$ & $4.04 \mathrm{E}+01$ & $4.78 E-06$ \\
\hline$I-129$ & $1.12 \mathrm{E}-02$ & 1. $26 \mathrm{E}-02$ & 1.26E-02 & $1.26 \mathrm{E}-02$ & $1.26 E-02$ & $1.15 \mathrm{E}-02$ \\
\hline $\mathrm{Kr}-85$ & $4.42 \mathrm{E}+02$ & $4.95 \mathrm{E}+02$ & $5.29 \mathrm{E}+02$ & $5.29 \mathrm{E}+02$ & $5.29 E+02$ & $4.14 \mathrm{E}+02$ \\
\hline $\mathrm{Np}-237$ & $1.12 \mathrm{E}-01$ & $1.35 \mathrm{E}-01$ & $1.39 \mathrm{E}-01$ & $1.39 \mathrm{E}-01$ & $1.39 E-01$ & $1.12 \mathrm{E}-01$ \\
\hline $\mathrm{Np}-239$ & $6.85 \mathrm{E}+00$ & $1.28 \mathrm{E}+01$ & $6.81 \mathrm{E}+00$ & $6.81 \mathrm{E}+00$ & $6.81 E+00$ & $7.30 \mathrm{E}+00$ \\
\hline $\mathrm{Pm}-147$ & $1.66 \mathrm{E}+01$ & $1.90 \mathrm{E}+01$ & $2.02 \mathrm{E}+01$ & $2.02 \mathrm{E}+01$ & $2.02 \mathrm{E}+01$ & $1.67 \mathrm{E}+01$ \\
\hline $\mathrm{Pu}-238$ & $7.47 \mathrm{E}+02$ & $1.21 \mathrm{E}+03$ & $9.32 \mathrm{E}+02$ & $9.32 \mathrm{E}+02$ & $9.32 \mathrm{E}+02$ & $9.55 \mathrm{E}+02$ \\
\hline Pu-239 & $1.16 \mathrm{E}+02$ & $9.78 \mathrm{E}+01$ & $1.25 \mathrm{E}+02$ & $1.25 \mathrm{E}+02$ & $1.25 E+02$ & $1.23 E+02$ \\
\hline $\mathrm{Pu}-240$ & $1.96 \mathrm{E}+02$ & $1.93 \mathrm{E}+02$ & $2.12 \mathrm{E}+02$ & $2.12 \mathrm{E}+02$ & $2.12 \mathrm{E}+02$ & $2.06 \mathrm{E}+02$ \\
\hline $\mathrm{Pu}-24 \mathrm{I}$ & $1.13 E+04$ & $1.25 E+04$ & $1.21 E+04$ & $1.21 E+04$ & $1.21 E+04$ & $1.21 \mathrm{E}+04$ \\
\hline $\mathrm{Pu}-242$ & $6.76 \mathrm{E}-01$ & $9.39 \mathrm{E}-01$ & $6.84 \mathrm{E}-01$ & $6.84 \mathrm{E}-0 \mathrm{I}$ & $6.84 \mathrm{E}-01$ & $6.99 \mathrm{E}-01$ \\
\hline Rh-105 & $2.25 \mathrm{E}-04$ & $5.56 \mathrm{E}-04$ & $2.89 E-04$ & $2.89 \mathrm{E}-04$ & $2.89 E-04$ & $2.94 \mathrm{E}-04$ \\
\hline $\mathrm{Ru}-106$ & $2.25 \mathrm{E}-04$ & $5.53 E-04$ & $2.89 E-04$ & $2.89 \mathrm{E}-04$ & $2.89 E-04$ & $2.93 E-04$ \\
\hline $\mathrm{Sb}-125$ & $2.82 E+00$ & $3.90 \mathrm{E}+00$ & $3.16 \mathrm{E}+00$ & $3.16 E+00$ & $3.16 \mathrm{E}+00$ & $2.35 E+00$ \\
\hline $\mathrm{sm}-151$ & $9.88 \mathrm{E}+01$ & $9.53 \mathrm{E}+01$ & $1.10 \mathrm{E}+02$ & $1.10 \mathrm{E}+02$ & 1. $10 \mathrm{E}+02$ & $1.03 \mathrm{E}+02$ \\
\hline Sr-90 & $1.18 \mathrm{E}+04$ & $1.28 \mathrm{E}+04$ & $1.42 \mathrm{E}+04$ & $1.42 \mathrm{E}+04$ & $1.42 \mathrm{E}+04$ & $1.16 \mathrm{E}+04$ \\
\hline TC-99 & $4.51 E+00$ & $4.86 E+00$ & $5.21 E+00$ & $5.21 E+00$ & $5.21 E+00$ & $4.66 \mathrm{E}+00$ \\
\hline $\mathrm{Te}-125 \mathrm{~m}$ & $6.87 E-01$ & $9.53 \mathrm{E}-01$ & 7. $70 \mathrm{E}-01$ & $7.70 \mathrm{E}-01$ & $7.70 \mathrm{E}-01$ & $5.72 \mathrm{E}-01$ \\
\hline $\mathrm{U}-234$ & $3.88 \mathrm{E}-01$ & $3.53 \mathrm{E}-01$ & $5.11 \mathrm{E}-01$ & $5.11 \mathrm{E}-01$ & 5. $11 \mathrm{E}-01$ & $4.44 \mathrm{E}-01$ \\
\hline $\mathrm{U}-235$ & $5.34 E-03$ & $2.88 \mathrm{E}-03$ & $6.91 \mathrm{E}-03$ & $6.91 \mathrm{E}-03$ & $6.91 E-03$ & $5.39 \mathrm{E}-03$ \\
\hline $\mathrm{U}-236$ & $8.16 \mathrm{E}-02$ & $7.95 \mathrm{E}-02$ & $1.07 \mathrm{E}-01$ & $1.07 \mathrm{E}-01$ & $1.07 \mathrm{E}-01$ & $8.29 \mathrm{E}-02$ \\
\hline$U-237$ & $2.77 E-01$ & $3.05 E-01$ & $2.97 \mathrm{E}-01$ & $2.97 \mathrm{E}-01$ & $2.97 E-0 I$ & $2.96 \mathrm{E}-01$ \\
\hline $\mathrm{U}-238$ & $1.19 \mathrm{E}-01$ & $9.71 \mathrm{E}-02$ & $1.27 E-01$ & $1.27 E-01$ & $1.27 E-01$ & $1.30 \mathrm{E}-01$ \\
\hline$Y-90$ & $1.18 \mathrm{E}+04$ & $1.28 \mathrm{E}+04$ & $1.42 \mathrm{E}+04$ & $1.42 \mathrm{E}+04$ & $1.42 \mathrm{E}+04$ & $1.16 \mathrm{E}+04$ \\
\hline TotaI & $7.31 E+04$ & $8.25 E+04$ & $8.47 E+04$ & $8.47 E+04$ & $8.47 E+04$ & $7.37 \mathrm{E}+04$ \\
\hline
\end{tabular}


Table C.4. LWR Spent Fuel Radioactivity as of Year 2030, Ci/Canister

\begin{tabular}{|c|c|c|c|c|c|c|}
\hline & \multicolumn{2}{|c|}{ Calvert Cliffs } & \multicolumn{3}{|c|}{ Point Beach } & \multirow{2}{*}{ Consolidated } \\
\hline Nuclide & D101 & D047 & $\mathrm{H}-07$ & $\mathrm{H}-12$ & $\mathrm{H}-25$ & \\
\hline Am-24I & $1.41 E+03$ & $1.46 \mathrm{E}+03$ & $1.49 \mathrm{E}+03$ & $1.49 E+03$ & $1.49 E+03$ & $1.51 E+03$ \\
\hline Am-242 & $3.12 \mathrm{E}+00$ & $3.91 E+00$ & $5.45 \mathrm{E}+00$ & $5.45 \mathrm{E}+00$ & $5.45 \mathrm{E}+00$ & $9.13 E+00$ \\
\hline$A m-242 m$ & $3.14 \mathrm{E}+00$ & $3.93 E+00$ & $5.48 \mathrm{E}+00$ & $5.48 E+00$ & $5.48 E+00$ & $9.17 \mathrm{E}+00$ \\
\hline $\mathrm{Am}-243$ & $6.83 E+00$ & 1. $28 \mathrm{E}+01$ & $6.80 E+00$ & $6.80 \mathrm{E}+00$ & $6.80 \mathrm{E}+00$ & $7.28 \mathrm{E}+00$ \\
\hline $\mathrm{Ba}-137 \mathrm{~m}$ & $1.06 \mathrm{E}+04$ & 1. $22 E+04$ & $1.23 E+04$ & $1.23 E+04$ & $1.23 E+04$ & $1.06 \mathrm{E}+04$ \\
\hline $\mathrm{cd}-113 \mathrm{~m}$ & $1.94 \mathrm{E}+00$ & $2.60 \mathrm{E}+00$ & $2.08 E+00$ & $2.08 E+00$ & $2.08 \mathrm{E}+00$ & $1.79 \mathrm{E}+00$ \\
\hline $\mathrm{Cm}-242$ & $2.58 \mathrm{E}+00$ & $3.23 E+00$ & $4.51 . E+00$ & $4.51 E+00$ & $4.51 \mathrm{E}+00$ & $7.55 \mathrm{E}+00$ \\
\hline $\mathrm{Cm}-243$ & $2.55 \mathrm{E}+00$ & $4.61 E+00$ & $2.05 \mathrm{E}+00$ & $2.05 E+00$ & $2.05 E+00$ & $3.21 \mathrm{E}+00$ \\
\hline $\mathrm{Cm}-244$ & $1.18 \mathrm{E}+02$ & $3.30 E+02$ & $1.16 \mathrm{E}+02$ & $1.16 \mathrm{E}+02$ & $1.16 \mathrm{E}+02$ & $1.32 \mathrm{E}+02$ \\
\hline $\mathrm{Co}-60$ & $1.29 E+00$ & $1.89 \mathrm{E}+00$ & $9.00 \mathrm{E}+00$ & $9.00 E+00$ & $9.00 \mathrm{E}+00$ & $1.41 E-04$ \\
\hline $\mathrm{Cs}-134$ & $2.67 E-03$ & $5.34 E-03$ & $3.32 \mathrm{E}-03$ & $3.32 \mathrm{E}-03$ & $3.32 \mathrm{E}-03$ & $2.80 \mathrm{E}-03$ \\
\hline $\mathrm{Cs}-137$ & $1.13 \mathrm{E}+04$ & $1.29 \mathrm{E}+04$ & $1.30 \mathrm{E}+04$ & $1.30 \mathrm{E}+04$ & $1.30 E+04$ & $1.12 \mathrm{E}+04$ \\
\hline $\mathrm{Eu}-154$ & $6.72 \mathrm{E}+01$ & $1.00 E+02$ & $7.76 \mathrm{E}+01$ & $7.76 \mathrm{E}+01$ & $7.76 \mathrm{E}+01$ & $7.01 \mathrm{E}+01$ \\
\hline $\mathrm{Eu}-155$ & $2.19 E+00$ & $3.47 \mathrm{E}+00$ & $2.56 E+00$ & $2.56 \mathrm{E}+00$ & $2.56 \mathrm{E}+00$ & $2.37 \mathrm{E}+00$ \\
\hline $\mathrm{H}-3$ & $1.51 \mathrm{E}-06$ & $4.53 \mathrm{E}-06$ & 1. $31 E+01$ & $1.31 \mathrm{E}+01$ & $1.31 \mathrm{E}+01$ & $1.55 E-06$ \\
\hline$I-129$ & $1.12 \mathrm{E}-02$ & $1.26 \mathrm{E}-02$ & $1.26 \mathrm{E}-02$ & $1.26 \mathrm{E}-02$ & $1.26 \mathrm{E}-02$ & $1.15 \mathrm{E}-02$ \\
\hline $\mathrm{Kr}-85$ & $1.21 \mathrm{E}+02$ & $1.36 \mathrm{E}+02$ & $1.45 \mathrm{E}+02$ & $1.45 \mathrm{E}+02$ & $1.45 \mathrm{E}+02$ & $1.14 \mathrm{E}+02$ \\
\hline$N p-237$ & $1.20 \mathrm{E}-01$ & $1.43 \mathrm{E}-01$ & $1.48 \mathrm{E}-01$ & $1.48 \mathrm{E}-01$ & $1.48 \mathrm{E}-01$ & $1.21 \mathrm{E}-01$ \\
\hline$N p-239$ & $6.83 E+00$ & $1.28 \mathrm{E}+01$ & $6.80 \mathrm{E}+00$ & $6.80 \mathrm{E}+00$ & $6.80 \mathrm{E}+00$ & $7.28 \mathrm{E}+00$ \\
\hline Pm-147 & $8.44 \mathrm{E}-02$ & $9.61 \mathrm{E}-02$ & $1.03 \mathrm{E}-01$ & $1.03 E-01$ & $1.03 \mathrm{E}-01$ & $8.49 \mathrm{E}-02$ \\
\hline Pu-238 & $6.38 \mathrm{E}+02$ & $1.04 \mathrm{E}+03$ & $7.96 \mathrm{E}+02$ & $7.96 \mathrm{E}+02$ & $7.96 \mathrm{E}+02$ & $8.16 \mathrm{E}+02$ \\
\hline Pu-239 & $1.16 \mathrm{E}+02$ & $9.77 \mathrm{E}+01$ & $1.25 \mathrm{E}+02$ & $1.25 \mathrm{E}+02$ & $1.25 E+02$ & $1.23 E+02$ \\
\hline Pu-240 & $1.96 \mathrm{E}+02$ & $1.93 \mathrm{E}+02$ & $2.12 \mathrm{E}+02$ & $2.12 \mathrm{E}+02$ & $2.12 \mathrm{E}+02$ & $2.06 E+02$ \\
\hline$P u-24 I$ & $4.31 E+03$ & $4.76 E+03$ & $4.63 \mathrm{E}+03$ & $4.63 E+03$ & $4.63 E+03$ & $4.61 E+03$ \\
\hline$P u-242$ & $6.76 \mathrm{E}-01$ & $9.39 \mathrm{E}-0 \mathrm{I}$ & $6.84 \mathrm{E}-01$ & $6.84 \mathrm{E}-01$ & $6.84 \mathrm{E}-01$ & $6.99 \mathrm{E}-01$ \\
\hline $\mathrm{Rh}-106$ & $2.39 \mathrm{E}-10$ & $5.89 \mathrm{E}-10$ & $3.07 E-10$ & $3.07 \mathrm{E}-10$ & $3.07 E-10$ & $3.12 \mathrm{E}-10$ \\
\hline Ru-106 & $2.39 \mathrm{E}-10$ & $5.89 \mathrm{E}-10$ & $3.07 E-10$ & $3.07 E-10$ & $3.07 \mathrm{E}-10$ & $3.12 \mathrm{E}-10$ \\
\hline$S b-125$ & $1.89 \mathrm{E}-02$ & $2.62 \mathrm{E}-02$ & $2.12 \mathrm{E}-02$ & $2.12 \mathrm{E}-02$ & $2.12 \mathrm{E}-02$ & $1.57 \mathrm{E}-02$ \\
\hline Sm-151 & $8.47 E+01$ & $8.17 \mathrm{E}+01$ & $9.44 \mathrm{E}+0 \mathrm{I}$ & $9.44 \mathrm{E}+01$ & $9.44 \mathrm{E}+0 \mathrm{I}$ & $8.79 \mathrm{E}+01$ \\
\hline $\mathrm{Sr}-90$ & $7.30 \mathrm{E}+03$ & $7.93 \mathrm{E}+03$ & $8.80 E+03$ & $8.80 \mathrm{E}+03$ & $8.80 \mathrm{E}+03$ & $7.19 \mathrm{E}+03$ \\
\hline$T C-99$ & $4.51 E+00$ & $4.86 \mathrm{E}+00$ & $5.21 E+00$ & $5.21 E+00$ & $5.21 \mathrm{E}+00$ & $4.66 \mathrm{E}+00$ \\
\hline $\mathrm{Te}-125 \mathrm{~m}$ & $4.61 E-03$ & $6.39 \mathrm{E}-03$ & $5.16 \mathrm{E}-03$ & $5.16 \mathrm{E}-03$ & $5.16 \mathrm{E}-03$ & $3.84 E-03$ \\
\hline $\mathrm{U}-234$ & $4.27 \mathrm{E}-01$ & $4.17 \mathrm{E}-01$ & $5.60 \mathrm{E}-01$ & $5.60 \mathrm{E}-01$ & $5.60 \mathrm{E}-01$ & $4.94 \mathrm{E}-01$ \\
\hline $\mathrm{U}-235$ & $5.34 \mathrm{E}-03$ & $2.88 \mathrm{E}-03$ & $6.91 E-03$ & $6.91 E-03$ & $6.91 E-03$ & $5.39 \mathrm{E}-03$ \\
\hline $\mathrm{U}-236$ & $8.17 \mathrm{E}-02$ & $7.97 \mathrm{E}-02$ & $1.07 \mathrm{E}-01$ & $1.07 \mathrm{E}-01$ & $1.07 \mathrm{E}-01$ & $8.30 \mathrm{E}-02$ \\
\hline $\mathrm{U}-237$ & $1.06 \mathrm{E}-01$ & $1.17 \mathrm{E}-01$ & $1.14 \mathrm{E}-01$ & $1.14 \mathrm{E}-01$ & $1.14 \mathrm{E}-01$ & $1.13 \mathrm{E}-01$ \\
\hline $\mathrm{U}-238$ & $1.19 \mathrm{E}-01$ & $9.71 \mathrm{E}-02$ & $1.27 \mathrm{E}-01$ & $1.27 \mathrm{E}-01$ & $1.27 \mathrm{E}-01$ & $1.30 \mathrm{E}-01$ \\
\hline$Y-90$ & $7.31 \mathrm{E}+03$ & $7.94 \mathrm{E}+03$ & $8.80 \mathrm{E}+03$ & $8.80 \mathrm{E}+03$ & $8.80 \mathrm{E}+03$ & $7.19 \mathrm{E}+03$ \\
\hline Total & $4.36 E+04$ & $4.91 E+04$ & $5.07 E+04$ & $5.07 E+04$ & $5.07 \mathrm{E}+04$ & $4.39 E+04$ \\
\hline
\end{tabular}


Table C.5. LWR Spent Fuel Radioactivity as of June 2100, Ci/Canister

\begin{tabular}{|c|c|c|c|c|c|c|}
\hline \multirow[b]{2}{*}{ Nuclide } & \multicolumn{2}{|c|}{ Calvert Cliffs } & \multicolumn{3}{|c|}{ Point Beach } & \multirow{2}{*}{ Consolidated } \\
\hline & D101 & D047 & $\mathrm{H}-07$ & $\mathrm{H}-12$ & $\mathrm{H}-25$ & \\
\hline Am-241 & $1.39 E+03$ & $1.44 \mathrm{E}+03$ & $1.47 \mathrm{E}+03$ & $1.47 \mathrm{E}+03$ & $1.47 \mathrm{E}+03$ & $1.48 \mathrm{E}+03$ \\
\hline$A m-242$ & $2.27 \mathrm{E}+00$ & $2.84 E+00$ & $3.96 \mathrm{E}+00$ & $3.96 \mathrm{E}+00$ & $3.96 E+00$ & $6.63 E+00$ \\
\hline$A m-242 m$ & $2.28 \mathrm{E}+00$ & $2.85 E+00$ & $3.98 \mathrm{E}+00$ & $3.98 \mathrm{E}+00$ & $3.98 E+00$ & $6.67 \mathrm{E}+00$ \\
\hline $\mathrm{Am}-243$ & $6.79 \mathrm{E}+00$ & $1.27 \mathrm{E}+01$ & $6.75 \mathrm{E}+00$ & $6.75 \mathrm{E}+00$ & $6.75 E+00$ & $7.23 \mathrm{E}+00$ \\
\hline $\mathrm{Ba}-137 \mathrm{~m}$ & $2.11 E+03$ & $2.41 E+03$ & $2.44 \mathrm{E}+03$ & $2.44 E+03$ & $2.44 \mathrm{E}+03$ & $2.10 \mathrm{E}+03$ \\
\hline $\mathrm{cd}-113 \mathrm{~m}$ & $6.96 \mathrm{E}-02$ & $9.36 \mathrm{E}-02$ & $7.48 \mathrm{E}-02$ & $7.48 \mathrm{E}-02$ & $7.48 \mathrm{E}-02$ & $6.44 \mathrm{E}-02$ \\
\hline $\mathrm{Cm}-242$ & $1.88 E+00$ & $2.35 E+00$ & $3.28 \mathrm{E}+00$ & $3.28 \mathrm{E}+00$ & $3.28 \mathrm{E}+00$ & $5.49 \mathrm{E}+00$ \\
\hline $\mathrm{Cm}-243$ & $4.65 \mathrm{E}-01$ & $8.40 E-01$ & $3.73 \mathrm{E}-01$ & $3.73 \mathrm{E}-01$ & $3.73 \mathrm{E}-01$ & $5.85 \mathrm{E}-01$ \\
\hline $\mathrm{Cm}-244$ & $8.12 E+00$ & $2.26 \mathrm{E}+01$ & $7.95 \mathrm{E}+00$ & $7.95 \mathrm{E}+00$ & $7.95 \mathrm{E}+00$ & $9.04 \mathrm{E}+00$ \\
\hline $\mathrm{Co}-60$ & $1.29 \mathrm{E}-04$ & $1.90 \mathrm{E}-04$ & $9.02 \mathrm{E}-04$ & $9.02 \mathrm{E}-04$ & $9.02 \mathrm{E}-04$ & 1.41E-08 \\
\hline Cs -134 & $1.61 \mathrm{E}-13$ & $3.22 \mathrm{E}-13$ & $2.00 E-13$ & $2.00 E-13$ & $2.00 \mathrm{E}-13$ & $1.69 \mathrm{E}-13$ \\
\hline Cs -137 & $2.23 E+03$ & $2.55 E+03$ & $2.58 \mathrm{E}+03$ & $2.58 \mathrm{E}+03$ & $2.58 \mathrm{E}+03$ & $2.22 \mathrm{E}+03$ \\
\hline$E u-154$ & $2.38 \mathrm{E}-01$ & $3.55 \mathrm{E}-01$ & $2.75 \mathrm{E}-01$ & $2.75 \mathrm{E}-01$ & $2.75 \mathrm{E}-01$ & $2.49 \mathrm{E}-01$ \\
\hline Eu-155 & 1.23E-04 & $1.96 \mathrm{E}-04$ & $1.44 \mathrm{E}-04$ & $1.44 \mathrm{E}-04$ & $1.44 \mathrm{E}-04$ & $1.34 \mathrm{E}-04$ \\
\hline $\mathrm{H}-3$ & $2.96 \mathrm{E}-08$ & $8.90 \mathrm{E}-08$ & $2.58 \mathrm{E}-01$ & $2.58 \mathrm{E}-01$ & $2.58 \mathrm{E}-01$ & $3.06 \mathrm{E}-08$ \\
\hline$I-129$ & $1.12 \mathrm{E}-02$ & $1.26 \mathrm{E}-02$ & $1.26 \mathrm{E}-02$ & $1.26 \mathrm{E}-02$ & $1.26 \mathrm{E}-02$ & $1.15 \mathrm{E}-02$ \\
\hline $\mathrm{Kr}-85$ & $1.31 \mathrm{E}+00$ & $1.47 \mathrm{E}+00$ & $1.57 \mathrm{E}+00$ & $1.57 E+00$ & $1.57 \mathrm{E}+00$ & $1.23 \mathrm{E}+00$ \\
\hline $\mathrm{Np}-237$ & $1.53 \mathrm{E}-01$ & $1.77 \mathrm{E}-01$ & $1.82 \mathrm{E}-01$ & $1.82 \mathrm{E}-01$ & $1.82 \mathrm{E}-01$ & $1.56 \mathrm{E}-01$ \\
\hline $\mathrm{Np}-239$ & $6.79 E+00$ & $1.27 \mathrm{E}+01$ & $6.75 \mathrm{E}+00$ & $6.75 E+00$ & $6.75 \mathrm{E}+00$ & $7.23 \mathrm{E}+00$ \\
\hline Pm-147 & $7.84 \mathrm{E}-10$ & $8.92 \mathrm{E}-10$ & $9.52 \mathrm{E}-10$ & $9.52 \mathrm{E}-10$ & $9.52 \mathrm{E}-10$ & $7.89 \mathrm{E}-10$ \\
\hline Pu-238 & $3.68 \mathrm{E}+02$ & $5.98 \mathrm{E}+02$ & $4.60 \mathrm{E}+02$ & $4.60 \mathrm{E}+02$ & $4.60 \mathrm{E}+02$ & $4.72 \mathrm{E}+02$ \\
\hline Pu-239 & $1.16 \mathrm{E}+02$ & $9.75 \mathrm{E}+01$ & $1.25 \mathrm{E}+02$ & $1.25 \mathrm{E}+02$ & $1.25 \mathrm{E}+02$ & $1.23 E+02$ \\
\hline $\mathrm{Pu}-240$ & $1.95 \mathrm{E}+02$ & $1.93 \mathrm{E}+02$ & $2.11 E+0.2$ & $2.11 \mathrm{E}+02$ & $2.11 E+02$ & $2.05 \mathrm{E}+02$ \\
\hline $\mathrm{Pu}-241$ & $1.48 \mathrm{E}+02$ & $1.64 \mathrm{E}+02$ & $1.59 \mathrm{E}+02$ & $1.59 \mathrm{E}+02$ & $1.59 \mathrm{E}+02$ & $1.59 \mathrm{E}+02$ \\
\hline $\mathrm{Pu}-242$ & $6.76 \mathrm{E}-01$ & $9.39 \mathrm{E}-01$ & $6.84 \mathrm{E}-01$ & $6.84 \mathrm{E}-01$ & $6.84 \mathrm{E}-01$ & $6.99 \mathrm{E}-01$ \\
\hline $\mathrm{Rh}-106$ & $2.98 \mathrm{E}-31$ & 7. $33 \mathrm{E}-31$ & $3.82 \mathrm{E}-31$ & $3.82 \mathrm{E}-31$ & $3.82 \mathrm{E}-31$ & $3.89 E-31$ \\
\hline$R u-106$ & $2.98 E-3 I$ & $7.33 \mathrm{E}-31$ & $3.83 E-31$ & $3.83 \mathrm{E}-31$ & $3.83 E-31$ & $3.89 E-31$ \\
\hline $\mathrm{Sb}-125$ & $4.66 \mathrm{E}-10$ & $6.46 \mathrm{E}-10$ & $5.22 \mathrm{E}-10$ & $5.22 \mathrm{E}-10$ & $5.22 \mathrm{E}-10$ & $3.88 \mathrm{E}-10$ \\
\hline $\mathrm{Sm}-151$ & 4. $94 \mathrm{E}+01$ & $4.77 \mathrm{E}+01$ & $5.51 \mathrm{E}+01$ & $5.51 \mathrm{E}+01$ & $5.51 E+01$ & $5.12 \mathrm{E}+01$ \\
\hline$S r-90$ & $1.38 \mathrm{E}+03$ & $1.50 \mathrm{E}+03$ & $1.66 \mathrm{E}+03$ & $1.66 \mathrm{E}+03$ & $1.66 \mathrm{E}+03$ & $1.36 \mathrm{E}+03$ \\
\hline TC-99 & $4.51 \mathrm{E}+00$ & $4.86 \mathrm{E}+00$ & $5.21 \mathrm{E}+00$ & $5.21 \mathrm{E}+00$ & $5.21 \mathrm{E}+00$ & $4.66 \mathrm{E}+00$ \\
\hline $\mathrm{Te}-125 \mathrm{~m}$ & $1.14 \mathrm{E}-10$ & $1.58 \mathrm{E}-10$ & $1.27 \mathrm{E}-10$ & $1.27 \mathrm{E}-10$ & $1.27 \mathrm{E}-10$ & $9.47 \mathrm{E}-11$ \\
\hline $\mathrm{U}-234$ & $5.25 \mathrm{E}-01$ & $5.75 \mathrm{E}-01$ & $6.82 \mathrm{E}-01$ & $6.82 \mathrm{E}-01$ & $6.82 \mathrm{E}-01$ & $6.18 \mathrm{E}-01$ \\
\hline $\mathrm{U}-235$ & $5.35 \mathrm{E}-03$ & $2.89 \mathrm{E}-03$ & $6.92 \mathrm{E}-03$ & $6.92 \mathrm{E}-03$ & $6.92 E-03$ & $5.40 \mathrm{E}-03$ \\
\hline $\mathrm{U}-236$ & $8.21 \mathrm{E}-02$ & $8.01 \mathrm{E}-02$ & $1.08 \mathrm{E}-01$ & $1.08 \mathrm{E}-0 \mathrm{I}$ & $1.08 \mathrm{E}-01$ & $8.34 \mathrm{E}-02$ \\
\hline$U-237$ & $3.64 \mathrm{E}-03$ & $4.02 \mathrm{E}-03$ & $3.91 \mathrm{E}-03$ & $3.91 \mathrm{E}-03$ & $3.91 E-03$ & $3.90 \mathrm{E}-03$ \\
\hline $\mathrm{U}-238$ & $1.19 \mathrm{E}-01$ & $9.71 \mathrm{E}-02$ & $1.27 \mathrm{E}-01$ & $1.27 \mathrm{E}-01$ & $1.27 \mathrm{E}-01$ & 1. $30 \mathrm{E}-01$ \\
\hline $\mathrm{Y}-90$ & $1.38 \mathrm{E}+03$ & $1.50 \mathrm{E}+03$ & $1.66 \mathrm{E}+03$ & $1.66 \mathrm{E}+03$ & $1.66 \mathrm{E}+03$ & $1.36 \mathrm{E}+03$ \\
\hline Total & $9.40 \mathrm{E}+03$ & $1.06 \mathrm{E}+04$ & $1.09 \mathrm{E}+04$ & $1.09 \mathrm{E}+0.4$ & $1.09 \mathrm{E}+04$ & $9.59 \mathrm{E}+03$ \\
\hline
\end{tabular}


Table C.6. LWR Spent Fuel Mass as of June 1996, g/Canister

\begin{tabular}{|c|c|c|c|c|c|c|}
\hline & \multicolumn{2}{|c|}{ Calvert Cliffs } & \multicolumn{3}{|c|}{ Point Beach } & \multirow{2}{*}{ Consolidated } \\
\hline Nuclide & D101 & D047 & $\mathrm{H}-07$ & $\mathrm{H}-12$ & $\mathrm{H}-25$ & \\
\hline Am-241 & $2.56 E+02$ & $2.54 \mathrm{E}+02$ & $2.68 \mathrm{E}+02$ & $2.68 \mathrm{E}+02$ & $2.68 \mathrm{E}+02$ & $2.75 \mathrm{E}+02$ \\
\hline $\mathrm{Am}-242$ & $4.51 E-06$ & $5.64 \mathrm{E}-06$ & $7.87 \mathrm{E}-06$ & $7.87 E-06$ & $7.87 \mathrm{E}-06$ & 1. $32 \mathrm{E}-05$ \\
\hline$A m-242 m$ & $3.77 \mathrm{E}-01$ & $4.72 \mathrm{E}-01$ & $6.58 \mathrm{E}-01$ & $6.58 \mathrm{E}-01$ & $6.58 \mathrm{E}-01$ & $1.10 \mathrm{E}+00$ \\
\hline$A m-243$ & $3.44 E+01$ & $6.43 \mathrm{E}+01$ & $3.42 \mathrm{E}+01$ & $3.42 \mathrm{E}+01$ & $3.42 \mathrm{E}+01$ & $3.66 \mathrm{E}+01$ \\
\hline $\mathrm{Ba}-137 \mathrm{~m}$ & 4. $34 E-05$ & $4.96 \mathrm{E}-05$ & $5.01 \mathrm{E}-05$ & $5.01 \mathrm{E}-05$ & $5.01 \mathrm{E}-05$ & 4. 32E-05 \\
\hline $\mathrm{Cd}-113 \mathrm{~m}$ & $4.48 \mathrm{E}-02$ & $6.03 \mathrm{E}-02$ & $4.82 \mathrm{E}-02$ & $4.82 \mathrm{E}-02$ & $4.82 \mathrm{E}-02$ & $4.15 \mathrm{E}-02$ \\
\hline $\mathrm{Cm}-242$ & $9.12 \mathrm{E}-04$ & $1.14 \mathrm{E}-03$ & $1.59 \mathrm{E}-03$ & $1.59 \mathrm{E}-03$ & $1.59 \mathrm{E}-03$ & $2.67 \mathrm{E}-03$ \\
\hline $\mathrm{Cm}-243$ & $1.13 \mathrm{E}-01$ & $2.04 \mathrm{E}-01$ & $9.06 \mathrm{E}-02$ & $9.06 \mathrm{E}-02$ & $9.06 \mathrm{E}-02$ & $1.42 \mathrm{E}-01$ \\
\hline $\mathrm{Cm}-244$ & $5.37 E+00$ & $1.50 \mathrm{E}+01$ & $5.26 \mathrm{E}+00$ & $5.26 \mathrm{E}+00$ & $5.26 E+00$ & $5.98 \mathrm{E}+00$ \\
\hline $\mathrm{Co}-60$ & $9.97 \mathrm{E}-02$ & $1.46 \mathrm{E}-01$ & $6.96 \mathrm{E}-01$ & $6.96 \mathrm{E}-01$ & $6.96 \mathrm{E}-01$ & $1.09 E-05$ \\
\hline $\mathrm{Cs}-134$ & $1.88 \mathrm{E}-01$ & $3.79 \mathrm{E}-01$ & $2.37 E-01$ & $2.37 \mathrm{E}-01$ & $2.37 \mathrm{E}-01$ & $1.98 \mathrm{E}-01$ \\
\hline $\mathrm{Cs}-137$ & $2.83 E+02$ & $3.24 \mathrm{E}+02$ & $3.28 \mathrm{E}+02$ & $3.28 \mathrm{E}+02$ & $3.28 \mathrm{E}+02$ & $2.82 \mathrm{E}+02$ \\
\hline $\mathrm{Eu}-154$ & $3.86 \mathrm{E}+00$ & $5.75 E+00$ & $4.45 \mathrm{E}+00$ & $4.45 \mathrm{E}+00$ & $4.45 \mathrm{E}+00$ & $4.02 \mathrm{E}+00$ \\
\hline$E u-155$ & $5.44 \mathrm{E}-01$ & $8.64 \mathrm{E}-01$ & $6.38 \mathrm{E}-01$ & $6.38 E-01$ & $6.38 \mathrm{E}-01$ & $5.90 \mathrm{E}-01$ \\
\hline $\mathrm{H}-3$ & $8.14 \mathrm{E}-03$ & $9.68 \mathrm{E}-03$ & $9.24 \mathrm{E}-03$ & $9.24 \mathrm{E}-03$ & $9.24 \mathrm{E}-03$ & $7.80 \mathrm{E}-03$ \\
\hline$I-129$ & $6.35 \mathrm{E}+01$ & $7.14 \mathrm{E}+01$ & $7.14 \mathrm{E}+01$ & $7.14 \mathrm{E}+01$ & $7.14 \mathrm{E}+01$ & $6.49 E+01$ \\
\hline $\mathrm{Kr}-85$ & $2.78 \mathrm{E}+00$ & $3.11 \mathrm{E}+00$ & $3.33 \mathrm{E}+00$ & $3.33 E+00$ & $3.33 \mathrm{E}+00$ & $2.61 \mathrm{E}+00$ \\
\hline Np -237 & $1.51 \mathrm{E}+02$ & $1.84 \mathrm{E}+02$ & $1.90 \mathrm{E}+02$ & $1.90 \mathrm{E}+02$ & $1.90 \mathrm{E}+02$ & 1. $51 \mathrm{E}+02$ \\
\hline $\mathrm{Np}-239$ & 2. $95 \mathrm{E}-05$ & $5.52 \mathrm{E}-0.5$ & $2.94 \mathrm{E}-05$ & $2.94 \mathrm{E}-05$ & $2.94 \mathrm{E}-05$ & $3.15 \mathrm{E}-05$ \\
\hline Pm-147 & 7. $24 \mathrm{E}-01$ & $8.25 E-01$ & $8.80 \mathrm{E}-01$ & $8.80 \mathrm{E}-01$ & $8.80 \mathrm{E}-01$ & $7.29 E-01$ \\
\hline$P u-238$ & $4.87 \mathrm{E}+01$ & $7.92 \mathrm{E}+01$ & $6.07 \mathrm{E}+01$ & $6.07 E+01$ & $6.07 E+01$ & $6.22 \mathrm{E}+01$ \\
\hline Pu-239 & $1.87 E+03$ & $1.57 \mathrm{E}+03$ & $2.01 \mathrm{E}+03$ & $2.01 E+03$ & $2.01 E+03$ & $1.99 \mathrm{E}+03$ \\
\hline $\mathrm{Pu}-240$ & $8.59 \mathrm{E}+02$ & $8.41 E+02$ & $9.29 \mathrm{E}+02$ & $9.29 E+02$ & $9.29 \mathrm{E}+02$ & $9.04 \mathrm{E}+02$ \\
\hline$P u-241$ & $2.15 \mathrm{E}+02$ & $2.37 E+02$ & $2.31 \mathrm{E}+02$ & $2.31 \mathrm{E}+02$ & $2.31 E+02$ & $2.30 \mathrm{E}+02$ \\
\hline Pu-242 & $1.77 \mathrm{E}+02$ & $2.46 \mathrm{E}+02$ & $1.79 \mathrm{E}+02$ & $1.79 \mathrm{E}+02$ & $1.79 \mathrm{E}+02$ & $1.83 \mathrm{E}+02$ \\
\hline $\mathrm{Rh}-106$ & $9.53 E-10$ & $2.37 E-09$ & $1.22 \mathrm{E}-09$ & $1.22 E-09$ & $1.22 \mathrm{E}-09$ & $1.25 \mathrm{E}-09$ \\
\hline $\mathrm{Ru}-106$ & $1.01 \mathrm{E}-03$ & $2.51 \mathrm{E}-03$ & $1.30 \mathrm{E}-03$ & $1.30 \mathrm{E}-03$ & $1.30 \mathrm{E}-03$ & $1.33 \mathrm{E}-03$ \\
\hline$S b-125$ & $9.05 \mathrm{E}-02$ & $1.26 \mathrm{E}-01$ & $1.01 E-01$ & $1.01 \mathrm{E}-01$ & $1.01 \mathrm{E}-01$ & $7.54 \mathrm{E}-02$ \\
\hline Sm-151 & $4.18 \mathrm{E}+00$ & $4.03 E+00$ & $4.66 \mathrm{E}+00$ & $4.66 \mathrm{E}+00$ & $4.66 \mathrm{E}+00$ & $4.34 \mathrm{E}+00$ \\
\hline$S r-90$ & $1.20 \mathrm{E}+02$ & $1.30 \mathrm{E}+02$ & $1.45 \mathrm{E}+02$ & $1.45 \mathrm{E}+02$ & $1.45 \mathrm{E}+02$ & $1.18 \mathrm{E}+02$ \\
\hline TC-99 & $2.66 \mathrm{E}+02$ & $2.86 \mathrm{E}+02$ & $3.07 E+02$ & $3.07 \mathrm{E}+02$ & $3.07 \mathrm{E}+02$ & $2.75 \mathrm{E}+02$ \\
\hline $\mathrm{Te}-125 \mathrm{~m}$ & $1.27 E-03$ & $1.76 \mathrm{E}-03$ & $1.42 \mathrm{E}-03$ & $1.42 \mathrm{E}-03$ & $1.42 \mathrm{E}-03$ & $1.05 \mathrm{E}-03$ \\
\hline $\mathrm{U}-234$ & $5.71 \mathrm{E}+01$ & $4.84 \mathrm{E}+01$ & $7.56 \mathrm{E}+01$ & $7.56 \mathrm{E}+01$ & $7.56 \mathrm{E}+01$ & $6.46 \mathrm{E}+01$ \\
\hline $\mathrm{U}-235$ & $2.47 \mathrm{E}+03$ & $1.33 E+03$ & $3.20 \mathrm{E}+03$ & $3.20 \mathrm{E}+03$ & $3.20 \mathrm{E}+03$ & $2.49 \mathrm{E}+03$ \\
\hline$U-236$ & $1.26 \mathrm{E}+03$ & $1.23 \mathrm{E}+03$ & $1.65 \mathrm{E}+03$ & $1.65 \mathrm{E}+03$ & $1.65 \mathrm{E}+03$ & $1.28 \mathrm{E}+03$ \\
\hline$U-237$ & $6.66 \mathrm{E}-06$ & $7.34 E-05$ & $7.14 \mathrm{E}-06$ & $7.14 \mathrm{E}-06$ & $7.14 \mathrm{E}-06$ & $7.12 \mathrm{E}-06$ \\
\hline$U-238$ & $3.53 E+05$ & $2.89 \mathrm{E}+05$ & $3.79 \mathrm{E}+05$ & $3.79 E+05$ & $3.79 E+05$ & $3.88 \mathrm{E}+05$ \\
\hline$Y-90$ & $3.01 E-02$ & $3.27 \mathrm{E}-02$ & $3.63 \mathrm{E}-02$ & $3.63 \mathrm{E}-02$ & $3.63 \mathrm{E}-02$ & $2.97 \mathrm{E}-02$ \\
\hline Total & $3.61 \mathrm{E}+05$ & $2.96 \mathrm{E}+05$ & $3.89 E+05$ & $3.89 \mathrm{E}+05$ & $3.89 E+05$ & $3.96 \mathrm{E}+05$ \\
\hline
\end{tabular}


Table C.7. LWR Spent Fuel Mass as of Year 2000, g/Canister

\begin{tabular}{|c|c|c|c|c|c|c|}
\hline \multirow[b]{2}{*}{ Nuclide } & \multicolumn{2}{|c|}{ Calvert Cliffs } & \multicolumn{3}{|c|}{ Point Beach } & \multirow{2}{*}{ Consolidated } \\
\hline & D101 & D047 & $\mathrm{H}-07$ & $\mathrm{H}-12$ & $\mathrm{H}-25$ & \\
\hline$A m-241$ & $2.93 \mathrm{E}+02$ & $2.94 \mathrm{E}+02$ & $3.07 E+02$ & $3.07 E+02$ & $3.07 E+02$ & $3.13 E+02$ \\
\hline $\mathrm{Am}-242$ & $4.43 E-06$ & $5.54 \mathrm{E}-06$ & $7.73 E-06$ & $7.73 \mathrm{E}-06$ & $7.73 E-06$ & $1.29 E-05$ \\
\hline $\mathrm{Am}-242 \mathrm{~m}$ & $3.70 E-01$ & $4.63 \mathrm{E}-01$ & $6.46 \mathrm{E}-01$ & $6.46 \mathrm{E}-01$ & $6.46 \mathrm{E}-01$ & $1.08 \mathrm{E}+00$ \\
\hline Am-243 & $3.44 E+01$ & $6.42 E+01$ & $3.42 \mathrm{E}+01$ & $3.42 \mathrm{E}+01$ & $3.42 \mathrm{E}+01$ & $3.66 \mathrm{E}+01$ \\
\hline $\mathrm{Ba}-137 \mathrm{~m}$ & $3.95 E-05$ & $4.52 E-05$ & $4.57 E-05$ & $4.57 E-05$ & $4.57 \mathrm{E}-05$ & $3.94 \mathrm{E}-05$ \\
\hline$c d-113 m$ & $3.71 E-02$ & $4.99 \mathrm{E}-02$ & $3.98 E-02$ & $3.98 \mathrm{E}-02$ & $3.98 \mathrm{E}-02$ & $3.43 E-02$ \\
\hline $\mathrm{Cm}-242$ & $8.97 E-04$ & $1.12 \mathrm{E}-03$ & 1. $57 \mathrm{E}-03$ & $1.57 E-03$ & $1.57 \mathrm{E}-03$ & $2.62 \mathrm{E}-03$ \\
\hline $\mathrm{Cm}-243$ & $1.03 E-01$ & $1.85 \mathrm{E}-01$ & $8.22 \mathrm{E}-02$ & $8.22 \mathrm{E}-02$ & $8.22 \mathrm{E}-02$ & $1.29 \mathrm{E}-01$ \\
\hline $\mathrm{Cm}-244$ & $4.61 E+00$ & $1.28 \mathrm{E}+01$ & $4.51 E+00$ & $4.51 E+00$ & $4.51 \mathrm{E}+00$ & $5.14 E+00$ \\
\hline Co- 60 & $5.89 E-02$ & $8.65 E-02$ & 4.11E-01 & 4.11E-01 & $4.11 \mathrm{E}-01$ & $6.44 \mathrm{E}-06$ \\
\hline Cs -134 & $4.91 E-02$ & $9.87 \mathrm{E}-02$ & $6.17 \mathrm{E}-02$ & $6.17 E-02$ & $6.17 \mathrm{E}-02$ & $5.16 \mathrm{E}-02$ \\
\hline Cs -137 & $2.58 \mathrm{E}+02$ & $2.96 \mathrm{E}+02$ & $2.99 \mathrm{E}+02$ & $2.99 \mathrm{E}+02$ & $2.99 \mathrm{E}+02$ & $2.57 \mathrm{E}+02$ \\
\hline$E u-154$ & $2.79 \mathrm{E}+00$ & $4.16 \mathrm{E}+00$ & $3.22 \mathrm{E}+00$ & $3.22 \mathrm{E}+00$ & $3.22 E+00$ & $2.91 E+00$ \\
\hline$E u-155$ & $3.11 \mathrm{E}-01$ & $4.94 \mathrm{E}-01$ & $3.65 E-01$ & $3.65 E-01$ & $3.65 \mathrm{E}-01$ & $3.37 E-01$ \\
\hline $\mathrm{E}-3$ & $8.46 \mathrm{E}-10$ & $2.54 E-09$ & $7.38 \mathrm{E}-03$ & $7.38 \mathrm{E}-03$ & $7.38 \mathrm{E}-03$ & $8.73 E-10$ \\
\hline$I-129$ & $6.35 \mathrm{E}+01$ & 7. $14 E+01$ & $7.14 E+01$ & $7.14 \mathrm{E}+01$ & $7.14 \mathrm{E}+01$ & $6.49 \mathrm{E}+01$ \\
\hline $\mathrm{Kr}-85$ & $2.15 E+00$ & $2.40 \mathrm{E}+00$ & $2.57 E+00$ & $2.57 \mathrm{E}+00$ & $2.57 \mathrm{E}+00$ & $2.01 \mathrm{E}+00$ \\
\hline$N p-237$ & $1.53 E+02$ & $1.86 \mathrm{E}+02$ & $1.92 \mathrm{E}+02$ & $1.92 \mathrm{E}+02$ & $1.92 \mathrm{E}+02$ & $1.53 \mathrm{E}+02$ \\
\hline $\mathrm{Np}-239$ & $2.95 \mathrm{E}-05$ & $5.52 \mathrm{E}-05$ & $2.94 \mathrm{E}-05$ & $2.94 \mathrm{E}-05$ & 2. $94 \mathrm{E}-05$ & $3.15 \mathrm{E}-05$ \\
\hline$P m-147$ & $2.52 \mathrm{E}-01$ & $2.87 \mathrm{E}-01$ & $3.06 \mathrm{E}-01$ & $3.06 \mathrm{E}-01$ & $3.06 \mathrm{E}-01$ & $2.53 \mathrm{E}-01$ \\
\hline $\mathrm{Pu}-238$ & 4. $72 \mathrm{E}+01$ & $7.68 \mathrm{E}+01$ & $5.89 \mathrm{E}+01$ & $5.89 \mathrm{E}+01$ & $5.89 E+01$ & $6.03 E+01$ \\
\hline $\mathrm{Pu}-239$ & $1.87 \mathrm{E}+03$ & $1.57 E+03$ & $2.01 E+03$ & $2.01 E+03$ & $2.01 E+03$ & $1.99 \mathrm{E}+03$ \\
\hline $\mathrm{Pu}-240$ & $8.59 \mathrm{E}+02$ & $8.43 E+02$ & $9.29 \mathrm{E}+02$ & $9.29 \mathrm{E}+02$ & $9.29 \mathrm{E}+02$ & $9.04 \mathrm{E}+02$ \\
\hline $\mathrm{Pu}-241$ & $1.77 \mathrm{E}+02$ & $1.96 \mathrm{E}+02$ & $1.90 \mathrm{E}+02$ & $1.90 \mathrm{E}+02$ & $1.90 \mathrm{E}+02$ & $1.90 \mathrm{E}+02$ \\
\hline Pu-242 & $1.77 E+02$ & $2.46 \mathrm{E}+02$ & $1.79 \mathrm{E}+02$ & $1.79 \mathrm{E}+02$ & $1.79 \mathrm{E}+02$ & $1.83 \mathrm{E}+02$ \\
\hline $\mathrm{Rh}-106$ & $6.09 \mathrm{E}-11$ & $1.51 \mathrm{E}-10$ & $7.81 \mathrm{E}-11$ & $7.81 \mathrm{E}-11$ & $7.81 \mathrm{E}-11$ & $7.97 \mathrm{E}-11$ \\
\hline $\mathrm{Ru}-106$ & $6.48 E-05$ & $1.61 \mathrm{E}-04$ & $8.31 E-05$ & $8.31 E-05$ & $8.31 E-05$ & $8.48 E-05$ \\
\hline$S b-125$ & $3.33 E-02$ & $4.61 E-02$ & $3.73 E-02$ & $3.73 \mathrm{E}-02$ & $3.73 E-02$ & $2.77 \mathrm{E}-02$ \\
\hline Sm-151 & $4.05 E+00$ & $3.91 \mathrm{E}+00$ & $4.52 \mathrm{E}+00$ & $4.52 \mathrm{E}+00$ & $4.52 \mathrm{E}+00$ & $4.20 \mathrm{E}+00$ \\
\hline Sr-90 & $1.09 \mathrm{E}+02$ & $1.19 \mathrm{E}+02$ & $1.31 E+02$ & $1.31 E+02$ & $1.31 E+02$ & $1.08 \mathrm{E}+02$ \\
\hline$T C-99$ & $2.66 \mathrm{E}+02$ & $2.86 \mathrm{E}+02$ & $3.07 \mathrm{E}+02$ & $3.07 \mathrm{E}+02$ & $3.07 \mathrm{E}+02$ & $2.75 \mathrm{E}+02$ \\
\hline $\mathrm{Te}-125 \mathrm{~m}$ & $4.65 \mathrm{E}-04$ & $6.46 \mathrm{E}-04$ & 5.21E-04 & $5.21 \mathrm{E}-04$ & $5.21 E-04$ & $3.88 E-04$ \\
\hline$U-234$ & $5.86 \mathrm{E}+01$ & $5.08 \mathrm{E}+01$ & $7.74 \mathrm{E}+01$ & 7. $74 \mathrm{E}+01$ & $7.74 E+01$ & $6.65 \mathrm{E}+01$ \\
\hline $\mathrm{U}-235$ & $2.47 E+03$ & $1.33 E+03$ & $3.20 \mathrm{E}+03$ & $3.20 E+03$ & $3.20 \mathrm{E}+03$ & $2.49 \mathrm{E}+03$ \\
\hline $\mathrm{U}-236$ & $1.26 E+03$ & $1.23 E+03$ & $1.65 \mathrm{E}+03$ & $1.65 E+03$ & $1.65 \mathrm{E}+03$ & $1.28 \mathrm{E}+03$ \\
\hline$U-237$ & $5.49 E-06$ & $6.05 \mathrm{E}-06$ & $5.89 \mathrm{E}-06$ & $5.89 \mathrm{E}-06$ & $5.89 \mathrm{E}-06$ & $5.88 \mathrm{E}-06$ \\
\hline $\mathrm{U}-238$ & $3.53 E+05$ & $2.89 \mathrm{E}+05$ & $3.79 \mathrm{E}+05$ & $3.79 \mathrm{E}+05$ & $3.79 E+05$ & $3.88 \mathrm{E}+05$ \\
\hline$Y-90$ & $2.74 \mathrm{E}-02$ & $2.98 \mathrm{E}-02$ & $3.30 \mathrm{E}-02$ & $3.30 \mathrm{E}-02$ & $3.30 \mathrm{E}-02$ & $2.70 \mathrm{E}-02$ \\
\hline Total & $3.61 E+05$ & $2.96 E+05$ & $3.89 E+05$ & $3.89 E+05$ & $3.89 E+05$ & $3.96 \mathrm{E}+05$ \\
\hline
\end{tabular}


Table C.8. LWR Spent Fuel Mass as of Year 2010, g/Canister

\begin{tabular}{|c|c|c|c|c|c|c|}
\hline \multirow[b]{2}{*}{ Nuclide } & \multicolumn{2}{|c|}{ Calvert Cliffs } & \multicolumn{3}{|c|}{ Point Beach } & \multirow{2}{*}{ Consolidated } \\
\hline & D101 & D047 & $\mathrm{H}-07$ & $\mathrm{H}-12$ & $\mathrm{H}-25$ & \\
\hline Am-241 & $3.55 E+02$ & $3.63 E+02$ & $3.74 E+02$ & $3.74 \mathrm{E}+02$ & $3.74 \mathrm{E}+02$ & $3.80 \mathrm{E}+02$ \\
\hline $\mathrm{Am}-242$ & $4.23 E-06$ & $5.30 \mathrm{E}-06$ & $7.39 \mathrm{E}-06$ & $7.39 E-06$ & $7.39 \mathrm{E}-06$ & $1.24 \mathrm{E}-05$ \\
\hline $\mathrm{Am}-242 \mathrm{~m}$ & $3.54 \mathrm{E}-01$ & $4.43 \mathrm{E}-01$ & $6.18 \mathrm{E}-01$ & $6.18 \mathrm{E}-01$ & $6.18 \mathrm{E}-01$ & $1.03 E+00$ \\
\hline$A m-243$ & $3.43 E+01$ & $6.42 \mathrm{E}+01$ & $3.42 \mathrm{E}+01$ & $3.42 \mathrm{E}+01$ & $3.42 \mathrm{E}+01$ & $3.66 \mathrm{E}+01$ \\
\hline $\mathrm{Ba}-137 \mathrm{~m}$ & $3.14 \mathrm{E}-05$ & $3.59 \mathrm{E}-0.5$ & $3.63 E-05$ & $3.63 \mathrm{E}-0.5$ & $3.63 E-05$ & $3.12 \mathrm{E}-05$ \\
\hline $\mathrm{Cd}-113 \mathrm{~m}$ & $2.31 E-02$ & $3.10 \mathrm{E}-02$ & $2.48 \mathrm{E}-02$ & $2.48 \mathrm{E}-02$ & $2.48 E-02$ & $2.13 \mathrm{E}-02$ \\
\hline $\mathrm{Cm}-242$ & $8.55 E-04$ & $1.07 E-03$ & $1.49 \mathrm{E}-03$ & $1.49 \mathrm{E}-03$ & $1.49 \mathrm{E}-03$ & $2.50 \mathrm{E}-03$ \\
\hline $\mathrm{Cm}-243$ & $8.04 \mathrm{E}-02$ & $1.45 \mathrm{E}-01$ & $6.44 \mathrm{E}-02$ & $6.44 \mathrm{E}-02$ & $6.44 \mathrm{E}-02$ & $1.01 \mathrm{E}-01$ \\
\hline $\mathrm{Cm}-244$ & $3.14 \mathrm{E}+00$ & $8.76 \mathrm{E}+00$ & $3.08 \mathrm{E}+00$ & $3.08 \mathrm{E}+00$ & $3.08 \mathrm{E}+00$ & $3.50 \mathrm{E}+00$ \\
\hline Co-60 & $1.58 \mathrm{E}-02$ & $2.32 \mathrm{E}-02$ & $1.10 \mathrm{E}-01$ & $1.10 \mathrm{E}-01$ & $1.10 \mathrm{E}-01$ & $1.73 E-06$ \\
\hline $\mathrm{Cs}-134$ & $1.70 \mathrm{E}-03$ & $3.42 \mathrm{E}-03$ & $2.14 \mathrm{E}-03$ & $2.14 \mathrm{E}-03$ & $2.14 \mathrm{E}-03$ & $1.79 E-03$ \\
\hline $\mathrm{Cs}-137$ & $2.05 \mathrm{E}+02$ & $2.35 \mathrm{E}+02$ & $2.37 \mathrm{E}+02$ & $2.37 E+02$ & $2.37 E+02$ & $2.04 E+02$ \\
\hline Eu-154 & $1.25 \mathrm{E}+00$ & $1.86 \mathrm{E}+00$ & $1.44 \mathrm{E}+00$ & $1.44 \mathrm{E}+00$ & $1.44 \mathrm{E}+00$ & $1.30 \mathrm{E}+00$ \\
\hline Eu-155 & $7.68 \mathrm{E}-02$ & $1.22 \mathrm{E}-01$ & $9.01 \mathrm{E}-02$ & $9.01 \mathrm{E}-02$ & $9.01 \mathrm{E}-02$ & $8.33 E-02$ \\
\hline $\mathrm{H}-3$ & $4.83 \mathrm{E}-10$ & $1.45 \mathrm{E}-09$ & $4.21 \mathrm{E}-03$ & $4.21 E-03$ & $4.21 E-03$ & $4.98 \mathrm{E}-10$ \\
\hline$I-129$ & $6.35 E+01$ & $7.14 \mathrm{E}+01$ & $7.14 \mathrm{E}+01$ & $7.14 \mathrm{E}+01$ & $7.14 \mathrm{E}+01$ & $6.49 \mathrm{E}+01$ \\
\hline $\mathrm{Kr}-85$ & $1.12 \mathrm{E}+00$ & $1.26 \mathrm{E}+00$ & $1.35 \mathrm{E}+00$ & $1.35 \mathrm{E}+00$ & $1.35 \mathrm{E}+00$ & $1.05 \mathrm{E}+00$ \\
\hline $\mathrm{Np}-237$ & $1.58 \mathrm{E}+02$ & $1.91 \mathrm{E}+02$ & $1.97 \mathrm{E}+02$ & $1.97 \mathrm{E}+02$ & $1.97 \mathrm{E}+02$ & $1.59 \mathrm{E}+02$ \\
\hline $\mathrm{Np}-239$ & $2.95 E-05$ & $5.52 \mathrm{E}-0.5$ & $2.93 \mathrm{E}-05$ & $2.93 E-05$ & $2.93 E-05$ & $3.15 \mathrm{E}-05$ \\
\hline Pm-147 & $1.79 \mathrm{E}-02$ & $2.04 \mathrm{E}-02$ & $2.18 \mathrm{E}-02$ & $2.18 \mathrm{E}-02$ & $2.18 \mathrm{E}-02$ & $1.80 \mathrm{E}-02$ \\
\hline $\mathrm{Pu}-238$ & $4.36 \mathrm{E}+01$ & 7. $10 \mathrm{E}+01$ & $5.44 \mathrm{E}+01$ & $5.44 \mathrm{E}+01$ & $5.44 \mathrm{E}+01$ & $5.58 \mathrm{E}+01$ \\
\hline $\mathrm{Pu}-239$ & $1.87 \mathrm{E}+03$ & $1.57 \mathrm{E}+03$ & $2.01 \mathrm{E}+03$ & $2.01 \mathrm{E}+03$ & $2.01 E+03$ & $1.99 \mathrm{E}+03$ \\
\hline $\mathrm{Pu}-240$ & $8.60 \mathrm{E}+02$ & $8.46 \mathrm{E}+02$ & $9.29 \mathrm{E}+02$ & $9.29 \mathrm{E}+02$ & $9.29 \mathrm{E}+02$ & $9.05 \mathrm{E}+02$ \\
\hline $\mathrm{Pu}-241$ & $1.10 \mathrm{E}+02$ & $1.21 \mathrm{E}+02$ & $1.18 \mathrm{E}+02$ & $1.18 \mathrm{E}+02$ & $1.18 \mathrm{E}+02$ & $1.17 \mathrm{E}+02$ \\
\hline Pu-242 & $1.77 \mathrm{E}+02$ & $2.46 \mathrm{E}+02$ & $1.79 \mathrm{E}+02$ & $1.79 \mathrm{E}+02$ & $1.79 \mathrm{E}+02$ & $1.83 \mathrm{E}+02$ \\
\hline $\mathrm{Rh}-106$ & $6.30 \mathrm{E}-14$ & $1.56 \mathrm{E}-13$ & $8.10 \mathrm{E}-14$ & $8.10 \mathrm{E}-14$ & $8.10 \mathrm{E}-14$ & $8.25 \mathrm{E}-14$ \\
\hline $\mathrm{Ru}-106$ & $6.71 \mathrm{E}-08$ & $1.65 \mathrm{E}-07$ & $8.62 \mathrm{E}-08$ & $8.62 \mathrm{E}-08$ & $8.62 \mathrm{E}-08$ & $8.76 \mathrm{E}-08$ \\
\hline $5 b-125$ & $2.72 \mathrm{E}-03$ & $3.78 \mathrm{E}-03$ & $3.05 \mathrm{E}-03$ & $3.05 E-03$ & $3.05 E-03$ & $2.27 \mathrm{E}-03$ \\
\hline Sm-151 & $3.75 \mathrm{E}+00$ & $3.62 \mathrm{E}+00$ & $4.18 \mathrm{E}+00$ & $4.18 \mathrm{E}+00$ & $4.18 \mathrm{E}+00$ & $3.89 \mathrm{E}+00$ \\
\hline$S r-90$ & $8.61 E+0.1$ & $9.35 \mathrm{E}+01$ & $1.04 \mathrm{E}+02$ & $1.04 \mathrm{E}+02$ & $1.04 E+02$ & $8.48 \mathrm{E}+01$ \\
\hline TC-99 & $2.66 \mathrm{E}+02$ & $2.86 \mathrm{E}+02$ & $3.07 E+02$ & $3.07 \mathrm{E}+02$ & $3.07 E+02$ & $2.75 \mathrm{E}+02$ \\
\hline $\mathrm{Te}-125 \mathrm{~m}$ & $3.81 E-05$ & $5.28 \mathrm{E}-05$ & $4.27 \mathrm{E}-05$ & $4.27 \mathrm{E}-05$ & $4.27 \mathrm{E}-05$ & $3.17 \mathrm{E}-05$ \\
\hline $\mathrm{U}-234$ & $6.21 E+01$ & $5.65 \mathrm{E}+01$ & $8.18 \mathrm{E}+01$ & $8.18 \mathrm{E}+01$ & $8.18 \mathrm{E}+01$ & 7. $10 \mathrm{E}+01$ \\
\hline $\mathrm{U}-235$ & $2.47 E+03$ & $1.33 \mathrm{E}+03$ & $3.20 \mathrm{E}+03$ & $3.20 \mathrm{E}+03$ & $3.20 \mathrm{E}+03$ & $2.49 \mathrm{E}+03$ \\
\hline $\mathrm{U}-236$ & $1.26 \mathrm{E}+03$ & $1.23 \mathrm{E}+03$ & $1.65 \mathrm{E}+03$ & $1.65 E+03$ & $1.65 \mathrm{E}+03$ & $1.28 \mathrm{E}+03$ \\
\hline$U-237$ & $3.39 E-06$ & $3.74 \mathrm{E}-06$ & $3.64 \mathrm{E}-06$ & $3.64 \mathrm{E}-06$ & $3.64 \mathrm{E}-06$ & $3.63 \mathrm{E}-06$ \\
\hline $\mathrm{U}-238$ & $3.53 \mathrm{E}+0.5$ & $2.89 \mathrm{E}+05$ & $3.79 \mathrm{E}+05$ & $3.79 \mathrm{E}+05$ & $3.79 \mathrm{E}+05$ & $3.88 \mathrm{E}+05$ \\
\hline$Y-90$ & $2.16 \mathrm{E}-02$ & $2.35 E-02$ & $2.60 \mathrm{E}-02$ & $2.60 \mathrm{E}-02$ & $2.60 \mathrm{E}-02$ & $2.13 \mathrm{E}-02$ \\
\hline Total & $3.61 E+05$ & $2.96 \mathrm{E}+05$ & $3.88 \mathrm{E}+05$ & $3.88 \mathrm{E}+05$ & $3.88 E+05$ & $3.96 \mathrm{E}+05$ \\
\hline
\end{tabular}


Table C.9. LWR Spent Fuel Mass as of Year $2030, \mathrm{~g} /$ Canister

\begin{tabular}{|c|c|c|c|c|c|c|}
\hline & \multicolumn{2}{|c|}{ Calvert Cliffs } & \multicolumn{3}{|c|}{ Point Beach } & \multirow{2}{*}{ Consolidated } \\
\hline Nuclide & D101 & D047 & $\mathrm{H}-07$ & $\mathrm{H}-12$ & $\mathrm{H}-25$ & \\
\hline $\mathrm{Am}-241$ & $4.10 \mathrm{E}+02$ & $4.25 E+02$ & $4.34 \mathrm{E}+02$ & $4.34 \mathrm{E}+02$ & $4.34 \mathrm{E}+02$ & $4.39 E+02$ \\
\hline$A m-242$ & $3.86 \mathrm{E}-06$ & $4.83 E-06$ & $6.74 \mathrm{E}-06$ & $6.74 E-06$ & $6.74 \mathrm{E}-06$ & $1.13 E-05$ \\
\hline$\underline{A m}-242 \mathrm{~m}$ & $3.23 \mathrm{E}-01$ & 4. $04 E-01$ & $5.64 \mathrm{E}-01$ & $5.64 \mathrm{E}-01$ & $5.64 \mathrm{E}-01$ & $9.44 \mathrm{E}-01$ \\
\hline $\mathrm{Am}-243$ & $3.43 \mathrm{E}+01$ & $6.41 E+01$ & $3.41 E+01$ & $3.41 E+01$ & $3.41 \mathrm{E}+01$ & $3.65 \mathrm{E}+01$ \\
\hline $\mathrm{Ba}-137 \mathrm{~m}$ & $1.98 \mathrm{E}-05$ & $2.26 \mathrm{E}-05$ & $2.29 \mathrm{E}-05$ & $2.29 E-05$ & $2.29 E-05$ & $1.97 E-05$ \\
\hline $\mathrm{cd}-113 \mathrm{~m}$ & $8.92 \mathrm{E}-03$ & 1.20E-02 & $9.58 \mathrm{E}-03$ & $9.58 \mathrm{E}-03$ & $9.58 \mathrm{E}-03$ & $8.25 E-03$ \\
\hline $\mathrm{Cm}-242$ & $7.81 E-04$ & $9.77 \mathrm{E}-04$ & $1.36 \mathrm{E}-03$ & $1.36 \mathrm{E}-03$ & $1.36 \mathrm{E}-03$ & $2.28 \mathrm{E}-03$ \\
\hline $\mathrm{Cm}-243$ & $4.94 \mathrm{E}-02$ & $8.93 E-02$ & $3.96 \mathrm{E}-02$ & $3.96 \mathrm{E}-02$ & $3.96 \mathrm{E}-02$ & $6.22 \mathrm{E}-02$ \\
\hline $\mathrm{Cm}-244$ & $1.46 \mathrm{E}+00$ & $4.08 E+00$ & $1.43 \mathrm{E}+00$ & $1.43 E+00$ & $1.43 E+00$ & $1.63 E+00$ \\
\hline $\mathrm{Co}-60$ & $1.14 \mathrm{E}-03$ & $1.67 \mathrm{E}-03$ & $7.94 \mathrm{E}-03$ & $7.94 \mathrm{E}-03$ & $7.94 \mathrm{E}-03$ & $1.24 \mathrm{E}-07$ \\
\hline $\mathrm{Cs}-134$ & $2.05 E-06$ & $4.13 \mathrm{E}-06$ & $2.56 \mathrm{E}-06$ & $2.56 \mathrm{E}-06$ & $2.56 \mathrm{E}-06$ & $2.16 \mathrm{E}-06$ \\
\hline $\mathrm{Cs}-137$ & $1.29 \mathrm{E}+02$ & $1.48 \mathrm{E}+02$ & $1.49 \mathrm{E}+02$ & $1.49 \mathrm{E}+02$ & $1.49 \mathrm{E}+02$ & $1.29 \mathrm{E}+02$ \\
\hline$E u-154$ & 2. $49 \mathrm{E}-01$ & $3.71 \mathrm{E}-01$ & $2.87 \mathrm{E}-01$ & $2.87 \mathrm{E}-01$ & $2.87 E-01$ & $2.60 \mathrm{E}-01$ \\
\hline Eu-155 & $4.69 \mathrm{E}-03$ & $7.46 \mathrm{E}-03$ & $5.50 \mathrm{E}-03$ & $5.50 \mathrm{E}-03$ & $5.50 \mathrm{E}-03$ & $5.09 \mathrm{E}-03$ \\
\hline $\mathrm{H}-3$ & $1.57 \mathrm{E}-10$ & $4.72 \mathrm{E}-10$ & $1.37 \mathrm{E}-03$ & $1.37 \mathrm{E}-03$ & $1.37 E-03$ & $1.62 \mathrm{E}-10$ \\
\hline$I-129$ & $6.35 \mathrm{E}+01$ & $7.14 \mathrm{E}+01$ & $7.14 \mathrm{E}+01$ & $7.14 \mathrm{E}+01$ & $7.14 \mathrm{E}+01$ & $6.49 E+01$ \\
\hline $\mathrm{Kr}-85$ & $3.09 \mathrm{E}-01$ & $3.46 \mathrm{E}-01$ & $3.70 \mathrm{E}-01$ & $3.70 \mathrm{E}-01$ & $3.70 \mathrm{E}-01$ & $2.89 \mathrm{E}-01$ \\
\hline Np -237 & 1. $71 \mathrm{E}+02$ & $2.04 \mathrm{E}+02$ & $2.10 \mathrm{E}+02$ & $2.10 \mathrm{E}+02$ & $2.10 \mathrm{E}+02$ & $1.72 \mathrm{E}+02$ \\
\hline Np-239 & $2.95 \mathrm{E}-05$ & $5.51 \mathrm{E}-05$ & $2.93 E-05$ & $2.93 E-05$ & $2.93 \mathrm{E}-0.5$ & $3.14 \mathrm{E}-05$ \\
\hline $\mathrm{Pm}-147$ & $9.09 \mathrm{E}-05$ & $1.04 \mathrm{E}-04$ & $1.10 \mathrm{E}-04$ & $1.10 E-04$ & $1.10 \mathrm{E}-04$ & $9.15 \mathrm{E}-05$ \\
\hline $\mathrm{Pu}-238$ & $3.73 E+01$ & $6.06 \mathrm{E}+01$ & $4.65 \mathrm{E}+01$ & $4.65 \mathrm{E}+01$ & $4.65 E+01$ & $4.77 \mathrm{E}+01$ \\
\hline Pu-239 & $1.87 E+03$ & $1.57 \mathrm{E}+03$ & $2.01 E+03$ & $2.01 E+03$ & $2.01 E+03$ & $1.98 \mathrm{E}+03$ \\
\hline $\mathrm{Pu}-240$ & $8.59 \mathrm{E}+02$ & $8.49 E+02$ & $9.29 \mathrm{E}+02$ & $9.29 \mathrm{E}+02$ & $9.29 E+02$ & $9.05 \mathrm{E}+02$ \\
\hline Pu-24I & $4.19 E+01$ & $4.62 \mathrm{E}+01$ & $4.49 \mathrm{E}+01$ & $4.49 \mathrm{E}+01$ & $4.49 \mathrm{E}+01$ & $4.48 \mathrm{E}+01$ \\
\hline Pu-242 & $1.77 \mathrm{E}+02$ & $2.46 \mathrm{E}+02$ & $1.79 \mathrm{E}+02$ & $1.79 \mathrm{E}+02$ & $1.79 \mathrm{E}+02$ & $1.83 E+02$ \\
\hline $\mathrm{Rh}-106$ & $6.71 E-20$ & $1.65 \mathrm{E}-19$ & $8.62 \mathrm{E}-20$ & $8.62 \mathrm{E}-20$ & $8.62 \mathrm{E}-20$ & $8.76 \mathrm{E}-20$ \\
\hline$R u-106$ & $7.14 \mathrm{E}-14$ & $1.76 \mathrm{E}-13$ & $9.17 E-14$ & $9.17 \mathrm{E}-14$ & $9.17 \mathrm{E}-14$ & $9.32 \mathrm{E}-14$ \\
\hline$S b-125$ & $1.83 E-05$ & $2.53 \mathrm{E}-0.5$ & $2.05 \mathrm{E}-05$ & $2.05 \mathrm{E}-0.5$ & $2.05 \mathrm{E}-05$ & $1.52 \mathrm{E}-05$ \\
\hline $\mathrm{Sm}-151$ & $3.22 \mathrm{E}+00$ & $3.10 \mathrm{E}+00$ & $3.59 \mathrm{E}+00$ & $3.59 \mathrm{E}+00$ & $3.59 \mathrm{E}+00$ & $3.34 \mathrm{E}+00$ \\
\hline Sr-90 & $5.35 E+01$ & $5.81 \mathrm{E}+01$ & $6.44 E+01$ & $6.44 \mathrm{E}+01$ & $6.44 \mathrm{E}+01$ & $5.27 \mathrm{E}+01$ \\
\hline TC-99 & $2.66 \mathrm{E}+02$ & $2.86 \mathrm{E}+02$ & $3.07 E+0.2$ & $3.07 \mathrm{E}+02$ & $3.07 \mathrm{E}+02$ & $2.75 \mathrm{E}+02$ \\
\hline $\mathrm{Te}-125 \mathrm{~m}$ & $2.55 E-07$ & $3.54 \mathrm{E}-07$ & $2.86 \mathrm{E}-07$ & $2.86 \mathrm{E}-07$ & $2.86 \mathrm{E}-07$ & $2.13 \mathrm{E}-07$ \\
\hline $\mathrm{U}-234$ & $6.84 \mathrm{E}+01$ & $6.67 \mathrm{E}+01$ & $8.97 \mathrm{E}+01$ & $8.97 \mathrm{E}+01$ & $8.97 E+01$ & $7.90 \mathrm{E}+01$ \\
\hline $\mathrm{U}-235$ & $2.47 E+03$ & 1. $33 E+03$ & $3.20 \mathrm{E}+03$ & $3.20 \mathrm{E}+03$ & $3.20 E+03$ & $2.49 E+03$ \\
\hline$U-236$ & $1.26 \mathrm{E}+03$ & $1.23 \mathrm{E}+03$ & $1.65 \mathrm{E}+03$ & $1.65 \mathrm{E}+03$ & $1.65 \mathrm{E}+03$ & $1.28 \mathrm{E}+03$ \\
\hline $\mathrm{U}-237$ & $1.30 \mathrm{E}-06$ & $1.43 \mathrm{E}-06$ & $1.39 \mathrm{E}-06$ & $1.39 E-06$ & $1.39 \mathrm{E}-06$ & $1.39 \mathrm{E}-06$ \\
\hline$U-238$ & $3.53 E+0.5$ & $2.89 \mathrm{E}+0.5$ & $3.79 \mathrm{E}+05$ & $3.79 \mathrm{E}+0.5$ & $3.79 E+05$ & $3.88 \mathrm{E}+05$ \\
\hline$Y-90$ & $1.34 \mathrm{E}-02$ & $1.46 \mathrm{E}-02$ & $1.61 \mathrm{E}-02$ & $1.61 \mathrm{E}-02$ & $1.61 \mathrm{E}-02$ & $1.32 \mathrm{E}-02$ \\
\hline Total & $3.61 E+05$ & $2.96 \mathrm{E}+05$ & $3.88 \mathrm{E}+05$ & $3.88 \mathrm{E}+05$ & $3.88 \mathrm{E}+05$ & $3.96 \mathrm{E}+05$ \\
\hline
\end{tabular}


Table C.10. LWR Spent Fuel Mass as of Year 2100, g/Canister

\begin{tabular}{|c|c|c|c|c|c|c|}
\hline & \multicolumn{2}{|c|}{ Calvert Cliffs } & \multicolumn{3}{|c|}{ Point Beach } & \multirow{2}{*}{ Consolidated } \\
\hline Nuclide & D101 & D047 & $\mathrm{H}-07$. & $\mathrm{H}-12$ & $\mathrm{H}-25$ & \\
\hline $\mathrm{Am}-241$ & $4.04 E+02$ & $4.21 E+02$ & $4.27 E+02$ & $4.27 E+02$ & $4.27 E+02$ & $4.33 \mathrm{E}+02$ \\
\hline Am-242 & $2.81 E-06$ & $3.51 \mathrm{E}-06$ & $4.90 \mathrm{E}-06$ & $4.90 E-06$ & $4.90 E-06$ & $8.21 E-06$ \\
\hline Am-242m & $2.35 E-01$ & $2.94 \mathrm{E}-01$ & $4.10 \mathrm{E}-01$ & $4.10 E-01$ & $4.10 E-01$ & $6.86 \mathrm{E}-01$ \\
\hline $\mathrm{Am}-243$ & $3.41 E+01$ & $6.37 \mathrm{E}+01$ & $3.39 E+01$ & $3.39 \mathrm{E}+01$ & $3.39 E+0 I$ & $3.63 E+01$ \\
\hline $\mathrm{Ba}-137 \mathrm{~m}$ & $3.92 E-06$ & $4.49 \mathrm{E}-06$ & $4.54 \mathrm{E}-06$ & $4.54 E-06$ & $4.54 \mathrm{E}-06$ & $3.90 \mathrm{E}-06$ \\
\hline $\mathrm{Cd}-113 \mathrm{~m}$ & $3.20 \mathrm{E}-04$ & $4.31 \mathrm{E}-04$ & $3.44 \mathrm{E}-04$ & $3.44 \mathrm{E}-04$ & $3.44 \mathrm{E}-04$ & $2.97 E-04$ \\
\hline $\mathrm{Cm}-242$ & $5.67 E-04$ & $7.10 \mathrm{E}-04$ & $9.91 \mathrm{E}-04$ & $9.91 \mathrm{E}-04$ & $9.91 \mathrm{E}-04$ & $1.66 \mathrm{E}-03$ \\
\hline $\mathrm{Cm}-243$ & $9.01 E-03$ & $1.63 \mathrm{E}-02$ & $7.22 \mathrm{E}-03$ & $7.22 \mathrm{E}-03$ & $7.22 \mathrm{E}-03$ & $1.13 \mathrm{E}-02$ \\
\hline $\mathrm{Cm}-244$ & $1.00 E-01$ & $2.80 \mathrm{E}-01$ & $9.83 \mathrm{E}-02$ & $9.83 \mathrm{E}-02$ & $9.83 \mathrm{E}-02$ & $1.12 \mathrm{E}-01$ \\
\hline Co-60 & $1.14 \mathrm{E}-07$ & $1.68 \mathrm{E}-07$ & $7.97 \mathrm{E}-07$ & $7.97 \mathrm{E}-07$ & $7.97 \mathrm{E}-07$ & $1.25 \mathrm{E}-11$ \\
\hline$C s-134$ & $1.24 \mathrm{E}-16$ & $2.49 E-16$ & $1.55 \mathrm{E}-16$ & $1.55 \mathrm{E}-16$ & $1.55 \mathrm{E}-16$ & 1. $30 \mathrm{E}-16$ \\
\hline $\mathrm{Cs}-137$ & $2.56 \mathrm{E}+01$ & $2.93 E+01$ & $2.96 \mathrm{E}+01$ & $2.96 \mathrm{E}+01$ & $2.96 \mathrm{E}+01$ & $2.55 E+01$ \\
\hline$E u-154$ & $8.82 \mathrm{E}-04$ & $1.31 \mathrm{E}-03$ & $1.02 \mathrm{E}-03$ & $1.02 \mathrm{E}-03$ & $1.02 \mathrm{E}-03$ & $9.20 \mathrm{E}-04$ \\
\hline$E u-155$ & $2.64 E-07$ & $4.21 E-07$ & $3.10 \mathrm{E}-07$ & $3.10 \mathrm{E}-07$ & $3.10 \mathrm{E}-07$ & $2.87 \mathrm{E}-07$ \\
\hline $\mathrm{H}-3$ & $3.09 \mathrm{E}-12$ & $9.28 \mathrm{E}-12$ & $2.69 \mathrm{E}-05$ & $2.69 E-05$ & $2.69 \mathrm{E}-05$ & $3.19 E-12$ \\
\hline$I-129$ & $6.35 \mathrm{E}+01$ & $7.14 \mathrm{E}+01$ & $7.14 \mathrm{E}+01$ & $7.14 \mathrm{E}+01$ & $7.14 \mathrm{E}+01$ & $6.49 \mathrm{E}+01$ \\
\hline $\mathrm{Kr}-85$ & $3.34 \mathrm{E}-03$ & $3.74 \mathrm{E}-03$ & $4.00 \mathrm{E}-03$ & $4.00 \mathrm{E}-03$ & $4.00 \mathrm{E}-03$ & $3.13 \mathrm{E}-03$ \\
\hline $\mathrm{Np}-237$ & $2.17 \mathrm{E}+02$ & $2.51 \mathrm{E}+02$ & $2.59 \mathrm{E}+02$ & $2.59 \mathrm{E}+02$ & $2.59 \mathrm{E}+02$ & $2.21 \mathrm{E}+02$ \\
\hline $\mathrm{Np}-239$ & $2.93 \mathrm{E}-0.5$ & $5.47 \mathrm{E}-05$ & $2.91 E-05$ & $2.91 \mathrm{E}-05$ & $2.91 \mathrm{E}-05$ & $3.12 \mathrm{E}-05$ \\
\hline$P m-147$ & $8.45 \mathrm{E}-13$ & $9.62 \mathrm{E}-13$ & $1.03 E-12$ & $1.03 \mathrm{E}-12$ & $1.03 \mathrm{E}-12$ & $8.50 \mathrm{E}-13$ \\
\hline $\mathrm{Pu}-238$ & $2.15 \mathrm{E}+01$ & $3.49 \mathrm{E}+01$ & $2.69 \mathrm{E}+01$ & $2.69 \mathrm{E}+01$ & $2.69 \mathrm{E}+01$ & $2.76 \mathrm{E}+01$ \\
\hline $\mathrm{Pu}-239$ & $1.87 \mathrm{E}+03$ & $1.57 \mathrm{E}+03$ & $2.00 \mathrm{E}+03$ & $2.00 \mathrm{E}+03$ & $2.00 \mathrm{E}+03$ & $1.98 \mathrm{E}+03$ \\
\hline $\mathrm{Pu}-240$ & $8.54 \mathrm{E}+02$ & $8.46 E+02$ & $9.24 E+02$ & $9.24 \mathrm{E}+02$ & $9.24 \mathrm{E}+02$ & $9.00 \mathrm{E}+02$ \\
\hline $\mathrm{Pu}-241$ & $1.44 \mathrm{E}+00$ & $1.59 \mathrm{E}+00$ & $1.55 \mathrm{E}+00$ & $1.55 \mathrm{E}+00$ & $1.55 \mathrm{E}+00$ & $1.54 \mathrm{E}+00$ \\
\hline $\mathrm{Pu}-242$ & $1.77 \mathrm{E}+02$ & $2.46 \mathrm{E}+02$ & $1.79 E+02$ & $1.79 \mathrm{E}+02$ & $1.79 \mathrm{E}+02$ & $1.83 \mathrm{E}+02$ \\
\hline $\mathrm{Rh}-106$ & 8. $36 E-41$ & $2.06 E-40$ & $1.07 \mathrm{E}-40$ & $1.07 \mathrm{E}-40$ & $1.07 \mathrm{E}-40$ & $1.09 \mathrm{E}-40$ \\
\hline $\mathrm{Ru}-106$ & $8.89 E-35$ & $2.19 \mathrm{E}-34$ & $1.14 \mathrm{E}-34$ & $1.14 \mathrm{E}-34$ & $1.14 \mathrm{E}-34$ & $1.16 \mathrm{E}-34$ \\
\hline$S b-125$ & $4.51 E-13$ & $6.25 E-13$ & $5.05 E-13$ & $5.05 \mathrm{E}-13$ & $5.05 \mathrm{E}-13$ & $3.75 \mathrm{E}-13$ \\
\hline $\mathrm{Sm}-151$ & $1.88 \mathrm{E}+00$ & $1.81 \mathrm{E}+00$ & $2.09 \mathrm{E}+00$ & $2.09 \mathrm{E}+00$ & $2.09 \mathrm{E}+00$ & $1.95 \mathrm{E}+00$ \\
\hline Sr-90 & $1.01 E+01$ & $1.10 \mathrm{E}+01$ & $1.22 \mathrm{E}+01$ & 1. $22 \mathrm{E}+01$ & $1.22 \mathrm{E}+01$ & $9.95 \mathrm{E}+00$ \\
\hline TC-99 & $2.66 \mathrm{E}+02$ & $2.86 E+02$ & $3.07 \mathrm{E}+02$ & $3.07 \mathrm{E}+02$ & $3.07 \mathrm{E}+02$ & $2.75 \mathrm{E}+02$ \\
\hline $\mathrm{Te}-125 \mathrm{~m}$ & $6.30 \mathrm{E}-15$ & $8.74 E-15$ & $7.07 \mathrm{E}-15$ & $7.07 \mathrm{E}-15$ & $7.07 \mathrm{E}-15$ & $5.25 \mathrm{E}-15$ \\
\hline $\mathrm{U}-234$ & $8.39 \mathrm{E}+01$ & $9.20 \mathrm{E}+01$ & $1.09 \mathrm{E}+02$ & $1.09 \mathrm{E}+02$ & $1.09 \mathrm{E}+02$ & $9.89 E+01$ \\
\hline $\mathrm{U}-235$ & $2.47 \mathrm{E}+03$ & $1.34 \mathrm{E}+03$ & $3.20 \mathrm{E}+03$ & $3.20 \mathrm{E}+03$ & $3.20 \mathrm{E}+03$ & $2.50 \mathrm{E}+03$ \\
\hline $\mathrm{U}-236$ & $1.27 \mathrm{E}+03$ & $1.24 \mathrm{E}+03$ & $1.66 \mathrm{E}+03$ & $1.66 \mathrm{E}+03$ & $1.66 \mathrm{E}+03$ & $1.29 \mathrm{E}+03$ \\
\hline $\mathrm{U}-237$ & $4.46 \mathrm{E}-08$ & $4.92 \mathrm{E}-08$ & $4.78 \mathrm{E}-08$ & $4.78 \mathrm{E}-08$ & $4.78 E-08$ & $4.77 E-08$ \\
\hline $\mathrm{U}-238$ & $3.53 E+0.5$ & $2.89 E+05$ & $3.79 E+05$ & $3.79 \mathrm{E}+05$ & $3.79 \mathrm{E}+05$ & $3.88 \mathrm{E}+05$ \\
\hline$Y-90$ & $2.53 \mathrm{E}-03$ & $2.75 \mathrm{E}-03$ & $3.05 \mathrm{E}-03$ & $3.05 \mathrm{E}-03$ & $3.05 \mathrm{E}-03$ & $2.50 \mathrm{E}-03$ \\
\hline Total & $3.61 E+05$ & $2.95 E+05$ & $3.88 E+05$ & $3.88 E+05$ & $3.88 E+05$ & $3.96 \mathrm{E}+05$ \\
\hline
\end{tabular}




\section{Distribution}

No. of

Copies

\section{OFFSITE}

12 DOE/Office of Scientific and Technical Information

Technical Resources

R. D. Carrell

723 The Parkway, Suite 200

Richland, WA 99352

\section{ONSITE}

\section{Westinghouse Hanford Company}

R. E. Broz

A. B. Carlson

A3-05

J. O. Dittmer

P. L. Scott

R. J. Smith

J. C. Van Keuren
R3-86

$\mathrm{N} 2-02$

$\mathrm{N} 2-02$

G1-11

A3-34
No. of

Copies

15 Pacific Northwest National Laboratory
A. L. Doherty
J. H. Jarrett
U. P. Jenquin (10)
S. D. Landsman
R. J. Migliore
K. A. Pauley

K8-34

P7-35

K8-34

P7-35

K8-34

K8-34 